\title{
European Radioisotope Thermoelectric Generators (RTGs) and Radioisotope Heater Units (RHUs) for Space Science and Exploration
}

\author{
Richard M. Ambrosi ${ }^{1}$ (D) Hugo Williams² • Emily Jane Watkinson ${ }^{1}$ • \\ Alessandra Barco $^{1}$ - Ramy Mesalam ${ }^{1}$. Tony Crawford ${ }^{1}$. Christopher Bicknell ${ }^{1}$. \\ Piyal Samara-Ratna ${ }^{1}$ - David Vernon ${ }^{1}$ - Nigel Bannister ${ }^{1}$ - Duncan Ross ${ }^{1}$. \\ Jonathan Sykes ${ }^{1}$ - Marie-Claire Perkinson ${ }^{3}$ - Christopher Burgess ${ }^{3}$. Colin Stroud ${ }^{4}$. \\ Stephen Gibson ${ }^{4} \cdot$ Alexander Godfrey $^{4} \cdot$ Robert G. Slater $^{4} \cdot$ Michael J. Reece $^{5}$. \\ Kan Chen $^{5} \cdot$ Kevin Simpson ${ }^{6} \cdot$ Richard Tuley $^{6}$ - Mark Sarsfield ${ }^{7}$ Tim P. Tinsley ${ }^{7}$. \\ Keith Stephenson $^{8}$ - Daniel Freis ${ }^{9} \cdot$ Jean-François Vigier ${ }^{9}$ - Rudy J.M. Konings ${ }^{9}$. \\ Christophe Fongarland $^{10}$ • Martin Libessart ${ }^{11}$. James Merrifield ${ }^{12}$. \\ Daniel P. Kramer ${ }^{13}$ • Jamie Byrne ${ }^{1}$ • Benjamin Foxcroft ${ }^{1}$
}

Received: 1 September 2019 / Accepted: 25 November 2019 / Published online: 11 December 2019 (C) The Author(s) 2019

Abstract Radioisotope power systems utilising americium-241 as a source of heat have been under development in Europe as part of a European Space Agency funded programme since 2009. The aim is to develop all of the building blocks that would enable Europe to

In Situ Exploration of the Ice Giants: Science and Technology

Edited by Olivier J. Mousis and David H. Atkinson

R.M. Ambrosi

rma8@leicester.ac.uk

1 Department of Physics and Astronomy, University of Leicester, University Rd, Leicester, LE1 7RH, UK

2 Department of Engineering, University of Leicester, University Rd, Leicester, LE1 7RH, UK

3 Airbus, Gunnels Wood Rd, Stevenage, SG1 2AS, UK

4 Lockheed Martin UK, Reddings Wood, Ampthill, Bedford MK45 2HD, UK

5 School of Engineering \& Materials Science, Queen Mary University of London, Mile End Rd, London, E1 4NS, UK

6 European Thermodynamics Ltd, 8 Priory Business Park, Kibworth, Leicestershire, LE8 0RX, UK

7 National Nuclear Laboratory, Sellafield, Seascale, Cumbria, CA20 1PG, UK

8 European Space Agency, ESTEC TEC-EP, Keplerlaan 1, 2201AZ Noordwijk, The Netherlands

9 European Commission, Joint Research Centre (JRC), P.O. Box 2340, 76125 Karlsruhe, Germany

10 ArianeGroup, 51-61 Route de Verneuil, 78133, Les Mereaux Cedex, France

11 ArianeGroup, Agence de Cadarache, Centre d'affaire Archimède, ZA Rourabeau, 13115 Saint Paul lez Durance, France

12 Fluid Gravity Engineering, 1 West St, Emsworth, PO10 7DX, UK

13 University of Dayton, 300 College Park, Dayton, OH 45469 USA 
launch and operate deep space and planetary missions in environments where use of solar power or alternative power generation technologies is challenging. Although some technical and policy work activity predate the ESA programme, the maturity of the technology has now reached a level that it can be incorporated in mission studies and roadmaps targeting the period from the mid 2020s onwards. This paper describes the state of the art in European radioisotope thermoelectric generators and radioisotope heater units. This paper includes: the evolution of the technical programme in detail; descriptions of the design; evolution of RTG and RHU devices from laboratory prototypes to more advanced fully functional systems; and experimental data obtained to date. This paper also outlines the technical challenges and multidisciplinary skills required to develop what is a world leading, original, significant and transformative technology solution for planetary science and exploration missions from the mid 2020s onwards.

Keywords Nuclear power $\cdot$ Radioisotope $\cdot$ Space mission $\cdot$ Space science $\cdot$ Exploration

\section{Introduction}

\subsection{Current and Historical Context}

Radioisotope Thermoelectric Generators (RTGs) and Radioisotope Heater Units (RHUs) are key enablers for exploration of outer planets, deep space and planetary surfaces (Masters et al. 2014; Arridge et al. 2014; Ambrosi et al. 2019). The historical development of RTGs and RHUs based on work carried out primarily in the USA and is described in detail in Bennett (2012), Hula (2015). The culmination of the pioneering work by Ken Jordan and John Birden at Mound Laboratories in the early 1950s (Birden and Jordan 1959), resulted in the development the General Purpose Heat Source (GPHS) and the GPHS-RTG, which powered the Cassini spacecraft (Hula 2015), New Horizons (Hula 2015) and a number of other flagship missions. More recently the GPHS has been used in the Multi-Mission RTG (MMRTG), which has been a very successful development for planetary surface applications (Hula 2015).

In order to address these challenges, $\mathrm{Pu}-238$ constant-rate production was re-started in the US with the aim of producing up to $1.5 \mathrm{~kg}$ per year by 2025 and the development of high temperature thermoelectrics including skutterudite based (Holgate et al. 2014) and zintl based generators (Caillat et al. 2006) has been underway for a number of years. The latter (zintls) are being considered as an option for a next-generation deep space RTG design. In addition, there has been some development work on reactor-based systems that would use Stirling conversion systems for electrical power generation (McLure 2018).

More recently, concerns in the US programme over long-term sustainability and challenges around demand for space nuclear power systems have also spurred a review of their launch safety framework. This review has also focused on how to make US space nuclear power solutions available to missions ranging from Discovery class through to Flagship missions. This has resulted in the release of a Presidential memorandum encapsulating some of these new principles (Trump 2019).

The European context of: an expanding exploration programme with a resurgence in interest in lunar missions; participation in the lunar "Gateway" programme as a stepping stone to Mars; and continued interest and preparation for future Mars missions (including sample return), presents a number of future opportunities for European space nuclear power technologies in challenging environments (Fig. 1). In addition, significant increased interest in 


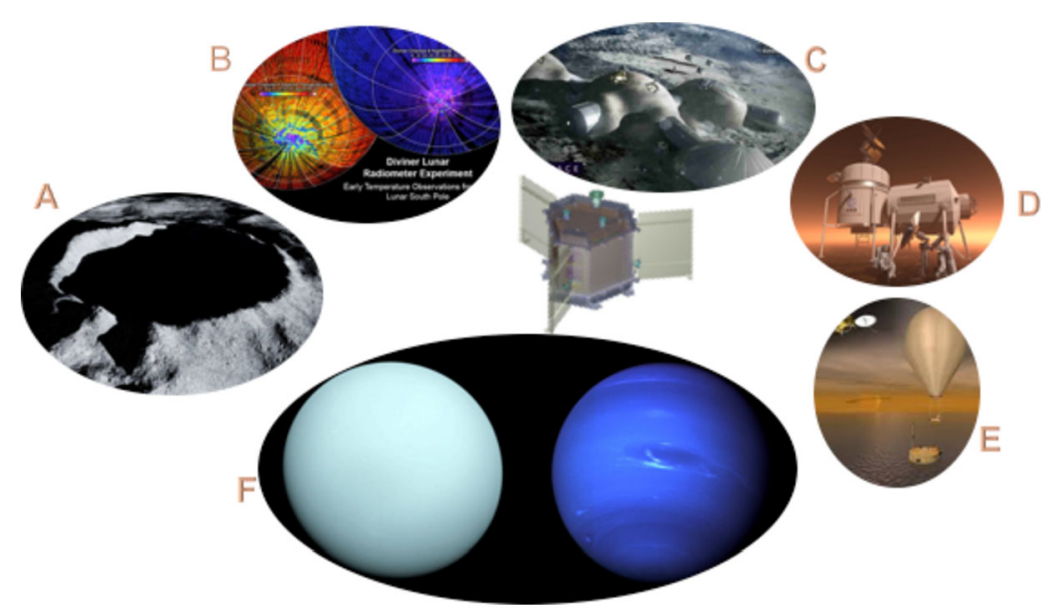

Fig. 1 (A) Permanently shadowed region on lunar surface with temperatures reaching $40 \mathrm{~K}$ as shown in (B). (C) Extensive human presence on the lunar surface planned. (D) Humans to Mars. (E) Tian surface and atmospheric probes. (F) Ice giants as future science mission targets. Images courtesy of ESA, NASA, JPL. Images from ESA are used under creative commons Creative Commons Attribution-ShareAlike 3.0 IGO (CC BY-SA 3.0 IGO)

deep space missions with the ice giants and their moons as targets (Fig. 1) also confirms the strategy of a European independent capability in alternatives to solar power including radioisotope power and heat. Although a European launch safety framework is still an important milestone to address in the coming years, the publication of the ESA Safety Policy on the Use of Nuclear Power Sources (ESA 2019) and ongoing work led by France on launch safety provides a greater degree of confidence that the launch of a European radioisotope power system in the second half of the 2020s is achievable.

\subsection{The ESA Radioisotope Power Systems Programme}

Radioisotope thermoelectric generators (RTG) and radioisotope heater units (RHU) are being developed in Europe as part of a European Space Agency (ESA) funded program. The development programme led by the University of Leicester has been based on a structured collaboration with industry. Aimed at enabling or significantly enhancing space science and exploration missions, these systems rely on the cost-effective production of americium-241 (in oxide form) for the fuel, an activity led by the National Nuclear Laboratory in the UK. The use of an iterative approach and the application of lean methodologies for the development these systems have been the focus of this technology program. Isotope containment architectures and, in the case of RTG systems, bismuth telluride based thermoelectric generators have been developed.

At the small end of the scale, the RHU configuration is based on a $3 \mathrm{~W}$ thermal power output unit purely for spacecraft thermal management applications. The RHU system has been designed, analysed and tested. In this paper a detailed description of the design and test results are provided. The paper is a review of the work carried out by the authors over a period of 10 years and references the contextual papers already in the literature.

The RTG design and overall system architecture has evolved since the University of Leicester began working on this project in 2010. The current RTG heat source configuration is designed to deliver between $200 \mathrm{~W}$ and $240 \mathrm{~W}$ of thermal power output (depending on the 
fuel form). A 5\% total system conversion efficiency and a modular scalable design imply that electrical power output can range between $10 \mathrm{~W}$ and $50 \mathrm{~W}$. Each RTG system could house up to 5 heat sources. An electrically-heated RTG system based on the $200 \mathrm{~W}$ heat source architecture has been designed, analysed and tested. A detailed summary of the evolution in RTG design and outputs of the programme to date is provided in this paper.

Stirling generator power conversion technology is being developed in the UK by Thales Alenia Space UK (Gilley et al. 2018). These systems aimed at producing around $100 \mathrm{~W}$ of electrical power per unit will not be reported in this paper as the work is being carried out is commercially sensitive.

Launch safety studies are being led by ArianeGroup in collaboration with a range of stakeholders in France and partners in the UK.

\section{Review of Americium-241 Based Fuel Production and Consolidation for RTGs and RHUs}

\subsection{Producing the Fuel}

In Europe, isotope selection studies have identified ${ }^{241} \mathrm{Am}$ as the isotope of choice for radioisotope-based systems. Americium fuel in oxide or ceramic form can be produced economically (when compared to producing ${ }^{238} \mathrm{Pu}$ in Europe) at a high isotopic purity by using a chemical separation method developed by the UK's National Nuclear Laboratory to extract it from stored separated plutonium produced during the reprocessing of civil fuel (Sarsfield et al. 2018). Spent civil reactor fuel can be reprocessed (Sarsfield et al. 2018) to extract all remaining $\mathrm{U}$ (mainly composed of ${ }^{238} \mathrm{U}$ ) and the $\mathrm{Pu}$ that has been produced. The $\mathrm{Pu}$ isotopes that are extracted are separated from the $U$ and other fission products and stored for future utilisation in mixed oxide fuel for power reactors. The $\mathrm{U}$ can flow back into the power generation cycle while the remaining fission products and other isotopes go into waste storage. Within the separated $\mathrm{Pu},{ }^{241} \mathrm{Am}$ builds up as a consequence of the beta decay of ${ }^{241} \mathrm{Pu}$ with a half-life of 14 years. The ingrowth of ${ }^{241} \mathrm{Am}$ implies continuity of supply without the requirement to use reactors or other infrastructure for production purposes.

The americium and plutonium purification by extraction or AMPPEX process developed by the National Nuclear Laboratory is described in detail by Sarsfield et al. (2018) and summarised in Fig. 2. The method for extracting the fuel is based on a silver catalyst assisted dissolution of both the $\mathrm{Pu}$ and Am isotopes in nitric acid, the separation of the Am while in solution, followed by oxalate precipitation of the Am and subsequent decomposition and calcination into an oxide ceramic powder (Sarsfield et al. 2018). It allows the separation of the ${ }^{241} \mathrm{Am}$ from the separated $\mathrm{Pu}$ and production of the ceramic form of the fuel $\mathrm{AmO}_{2}$. The production of the ceramic fuel and development of the fuel form is led by the UK's National Nuclear Laboratory supported by partners including: University of Leicester and Joint Research Centre (JRC) in Karlsruhe Germany.

Properties of $\mathrm{AmO}_{2}$ (and other oxides of Am including $\mathrm{Am}_{2} \mathrm{O}_{3}$ ) include: a thermal power output of $\sim 0.1 \mathrm{~W} / \mathrm{g}$; a 432-year half-life with a factor of approximately 4 reduction in power density when compared to ${ }^{238} \mathrm{Pu}$ based fuel used in the GPHS heat source $\left(\mathrm{PuO}_{2}\right)$; and a thermal conductivity of approximately $5 \mathrm{~W} / \mathrm{mK}$ reducing to about half that value for $\mathrm{Am}_{2} \mathrm{O}_{3}$ (Nishi et al. 2011). The melting point is around $2100{ }^{\circ} \mathrm{C}$, although $\mathrm{AmO}_{2}$ tends to decompose at high temperatures by losing oxygen (Konings et al. 2014).

A $50 \mathrm{~W}$ electric RTG system, for example, could therefore require approximately $10 \mathrm{~kg}$ of fuel. This is based on a 5\% system level conversion efficiency (Ambrosi et al. 2019) and a 


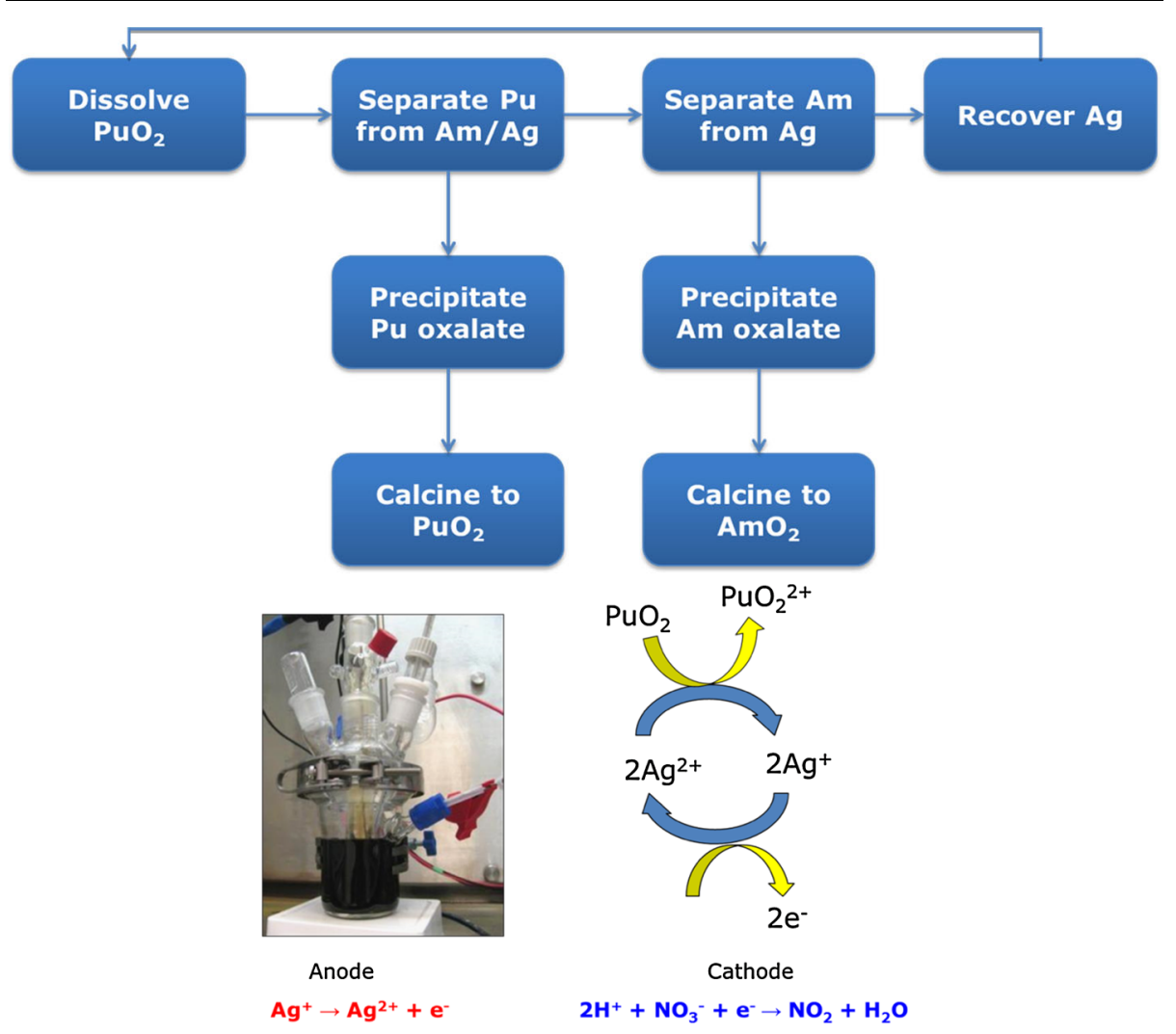

Fig. 2 Schematic of the AMPPEX process courtesy of National Nuclear Laboratory. The dissolution of the $\mathrm{Pu}$ in nitric acid is enhanced by utilising a silver II based solvent extraction method. The aqueous process allows the separation of ${ }^{241} \mathrm{Am}$ from the $\mathrm{Pu}$. The introduction of oxalic acid allows oxalate precipitation of the $\mathrm{Am}$ and subsequent calcination into $\mathrm{AmO}_{2}$. (Images above reprinted from Sarsfield et al. 2018; RightsLink, Elsevier License 4623650498499)

total thermal power output of $1 \mathrm{~kW}$ from $10 \mathrm{~kg}$ of fuel. Whereas a $3 \mathrm{~W}$ thermal RHU, which is likely to be the first European radioisotope power source flown in an ESA mission, would need approximately $30 \mathrm{~g}$ of fuel.

\subsection{Consolidating the Fuel}

An important part of the fuel production activity includes developing a suitable fuel form and consolidated structure, such as a pellet or disc, that has handling strength and that can be inserted into a heat source containment architecture (Watkinson et al. 2017a).

Americium oxides can have a range of metal to oxygen ratios from 1.5 to 2 (i.e. $\mathrm{Am}_{2} \mathrm{O}_{3}$ to $\mathrm{AmO}_{2}$ ) and the following tentative phase diagram (Fig. 3) outlines the various crystalline forms as a function of oxygen to metal ratio as well as temperature (Thiriet and Konings 2003).

A more up-to-date version of the diagram in Fig. 3, which highlights the inclusion of three main phases, rather than four, is provided by Epifano et al. (2017). In that paper, Epifano et al. state that the three main phases include the cubic or fluorite structure of the $\mathrm{AmO}_{2}$ and $\mathrm{AmO}_{2-x}$; a body centred cubic phase $\left(\mathrm{C}-\mathrm{AmO}_{1.61+x}\right)$, and the hexagonal $\mathrm{Am}_{2} \mathrm{O}_{3}$. 

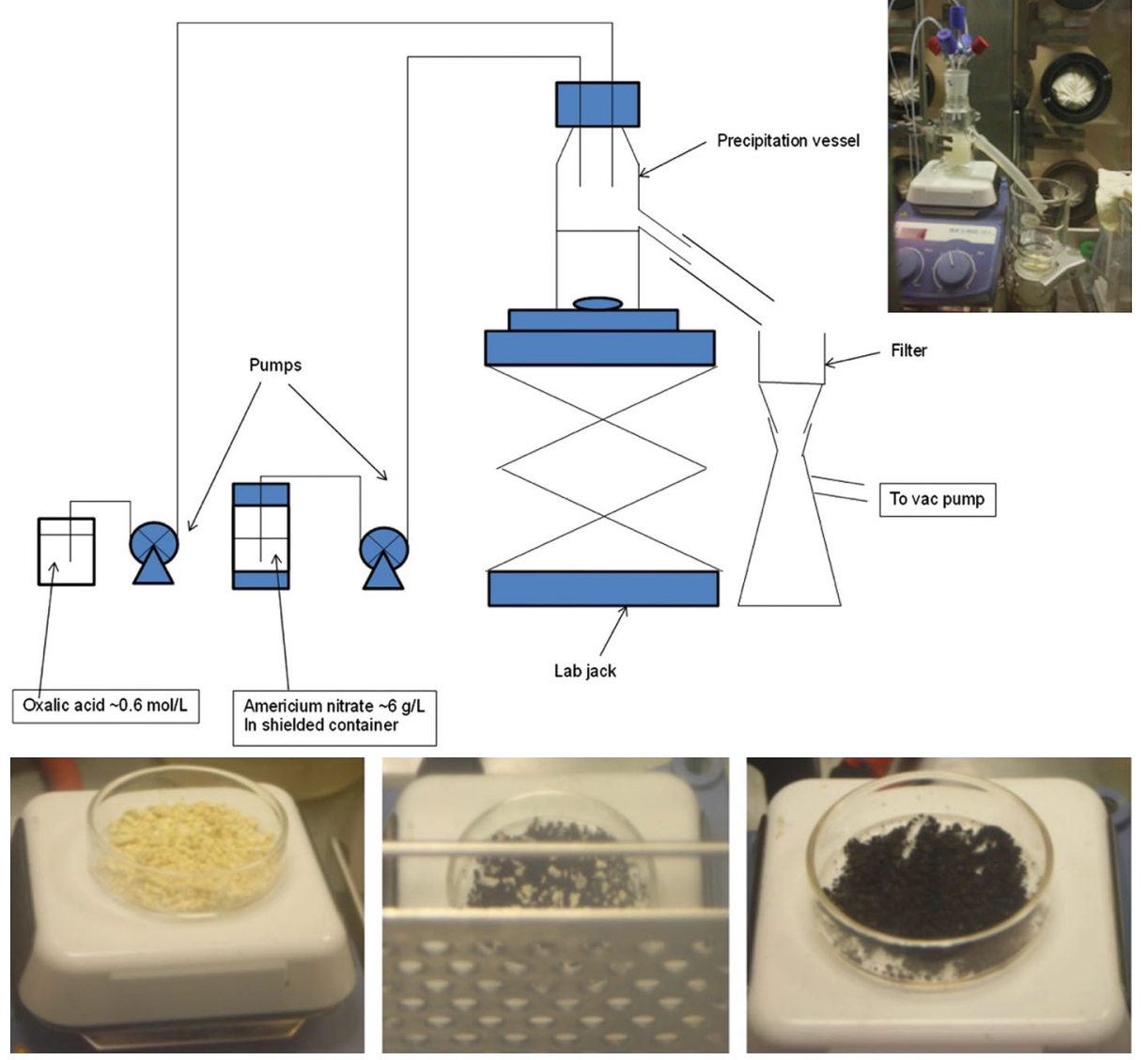

Fig. 2 (continued)

This updated work outlines that $\mathrm{Am}_{2} \mathrm{O}_{3}$ is stable at room temperature and that the phase transition outlined in early work by Thiriet and Konings does not occur; however, $\mathrm{Am}_{2} \mathrm{O}_{3}$ will readily oxidise.

As oxygen-to-metal ratios change from $\mathrm{AmO}_{2}$ to $\mathrm{Am}_{2} \mathrm{O}_{3}$, crystalline structure can also change from cubic to hexagonal. Studies have shown that when $\mathrm{AmO}_{2}$ is heated during consolidation or sintering of the ceramic fuel, depending on sintering environment and conditions, the fuel will tend to reduce, i.e. give up oxygen and form $\mathrm{Am}_{2} \mathrm{O}_{3}$. The final oxygen-tometal ratio depends on the atmosphere and temperature during the heating process (Chikalla and Eyring 1968; Lebreton et al. 2012). This is also highlighted by Sarsfield et al. (2017) and Epifano et al. (2017). Therefore, the focus for the ESA programme shifted to $\mathrm{Am}_{2} \mathrm{O}_{3}$ (Watkinson et al. 2017a) and more recently to a uranium-stabilised cubic americium oxide (Vigier et al. 2018) to produce a consolidated fuel form.

Research with surrogates of americium oxides carried out by Watkinson et al. (2017a, 2017b), Watkinson (2017), were useful in learning more about the production processes and consolidation of americium oxides while limiting contact with the radioactive material. Americium oxide surrogates (e.g. $\mathrm{Nd}_{2} \mathrm{O}_{3}$ for $\mathrm{Am}_{2} \mathrm{O}_{3}$ and $\mathrm{CeO}_{2}$ for $\mathrm{AmO}_{2}$ ) were recently 
Fig. 3 The tentative Am-O phase diagram by Thiriet and Konings (2003), which also illustrates data (circles) measured by Sari and Zamorani (1970). The $\mathrm{C}$ phase corresponds to cubic (Ia-3) $\mathrm{Am}_{2} \mathrm{O}_{3}$; the A phase to hexagonal $\mathrm{Am}_{2} \mathrm{O}_{3}$; the $\mathrm{C}^{\prime}$ phase to a higher temperature cubic (Ia-3) phase and all $\alpha$ phases to face centred cubic $\mathrm{AmO}_{2-(x / 2)}$. (Images above reprinted from Thiriet and Konings 2003; RightsLink, Elsevier License 4703021261997)

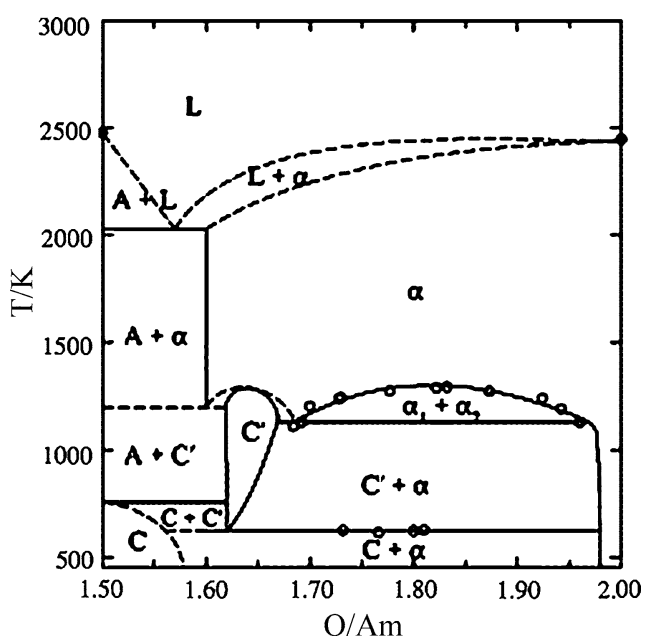

produced using two different techniques (continuous oxalate precipitation and calcination; and sol-gel) with the objective of creating particles with differing shapes or morphology (Watkinson et al. 2018).

$\mathrm{Nd}_{2} \mathrm{O}_{3}$ (like $\mathrm{Am}_{2} \mathrm{O}_{3}$ ) exhibits polymorphic properties, when produced from calcination of precursors at moderate temperature (i.e. $600{ }^{\circ} \mathrm{C}$ ). When heated at high temperatures (under sintering conditions) both are hexagonal, and they remain hexagonal when cooled down to room temperature. X-ray diffraction is therefore used routinely (as a function of temperature) to assess crystal structure changes in the ceramic powder to support sintering studies. The surrogate powders can be cold-pressed and sintered to assess the impact on pellet properties e.g. density, mechanical properties or integrity. The surrogate fuel studies have highlighted the importance of assessing the impact of co-precipitation conditions, particle shape (or morphology) and crystal structure (Watkinson et al. 2015; Sarsfield et al. 2017; Watkinson et al. 2017a, 2018) on the ability to meet fuel form requirements and will inform future research with the americium oxide fuel. Recent ESA studies have shown that the shape of the particles produced can have an impact on the ability to consolidate americiumbased ceramic fuels. Examples of the shape of particles that can be produced via various wet chemistry processes (Watkinson et al. 2018; Sarsfield et al. 2017) are shown in Fig. 4 and Fig. 5. In addition, these figures show the result that can be obtained from the sintering of surrogates of $\mathrm{AmO}_{2}$ and $\mathrm{Am}_{2} \mathrm{O}_{3}$ produced via different methods described in detail by Watkinson et al. (2017b).

Different sintering methods: cold-pressing and sintering or spark plasma sintering (Watkinson et al. 2017b, 2018; Kramer et al. 2018) have been tested. The first spark plasma sintering trials of $\mathrm{Nd}_{2} \mathrm{O}_{3}$ as a surrogate for $\mathrm{Am}_{2} \mathrm{O}_{3}$ are presented by Watkinson et al. (2017b). Varying sintering conditions can affect oxygen mobility in surrogates and are essential to our understanding stability of americium oxide based ceramic fuels. Greater control over environmental conditions (air, inert gas, reducing environment) and extended sintering periods of the former method, listed above, were traded off against the latter process, which was characterised by fast processing times and the ability to produce a variety of near net shape structures.

Among the various surrogate based studies, a Ce-Nd oxide solid solution study was carried out to investigate surrogates that could emulate $\mathrm{AmO}_{2-X}$ and help to understand the sub-stoichiometric behaviour of $\mathrm{AmO}_{2}$ (Watkinson et al. 2017a, 2018). An investigation 

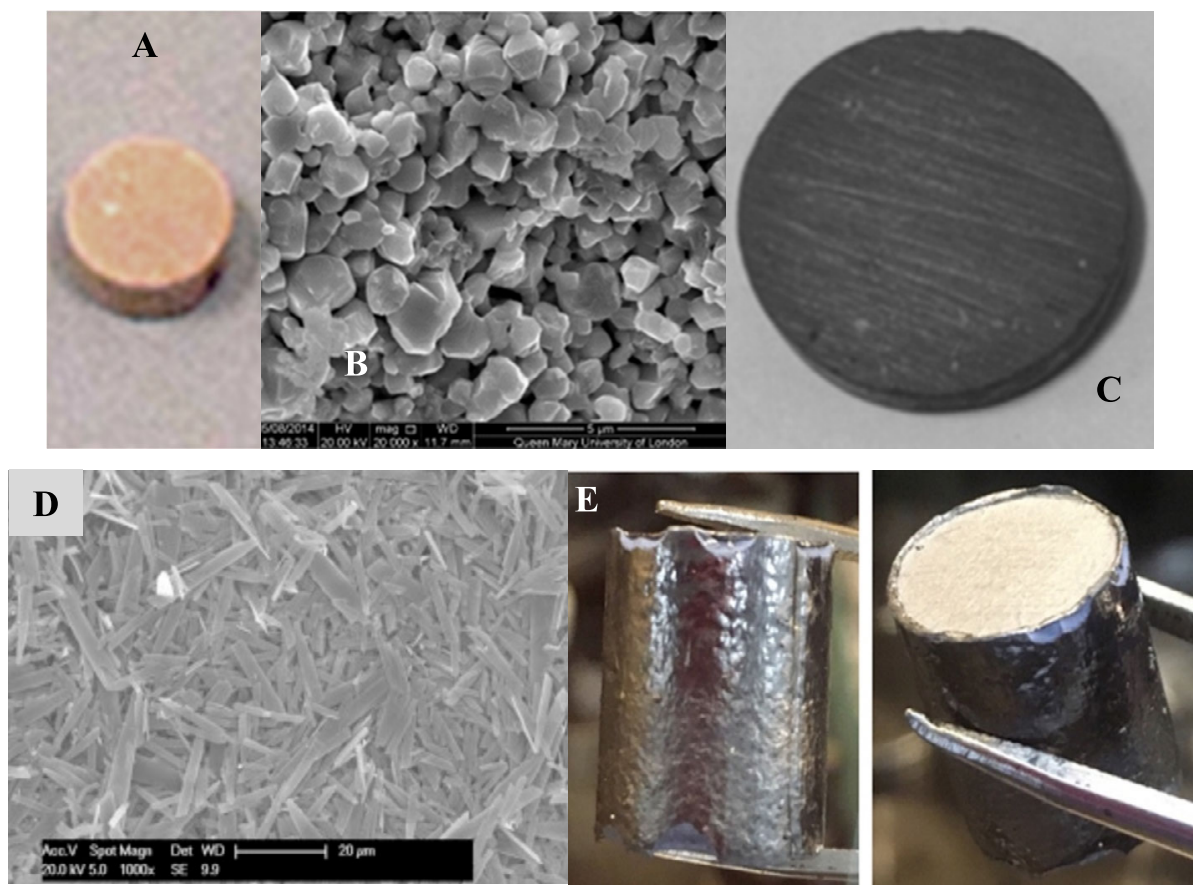

Fig. 4 (A) Consolidated ceria $\left(\mathrm{CeO}_{2}\right)$ pellet produced by the University of Dayton working with the University of Leicester using material produced via continuous oxalate precipitation and calcination (Watkinson et al. 2015). Ceria is a surrogate for $\mathrm{AmO}_{2}$. (B) Particle morphology of commercial $\mathrm{CeO}_{2}$, showing "potato-like" particles of order 1 micron in size. (C) Sintered commercial ceria produced by the University of Leicester and Queen Mary University of London using spark plasma sintering in a graphite die (Watkinson et al. 2017a). Disc is grey because it has lost some oxygen through reduction (Watkinson et al. 2017a). (D) Needle shaped particles of $\mathrm{Nd}_{2} \mathrm{O}_{3}$ produced by the University of Leicester using continuous precipitation and calcination process (Watkinson et al. 2017a). (E) Spark plasma sintered commercially sourced $\mathrm{Nd}_{2} \mathrm{O}_{3}$. (Some of the images above reprinted from Watkinson et al. (2017b); C. RightsLink Elsevier License 4660251270059 and also from Watkinson et al. (2017a); C. RightsLink Elsevier License 4623651311503)
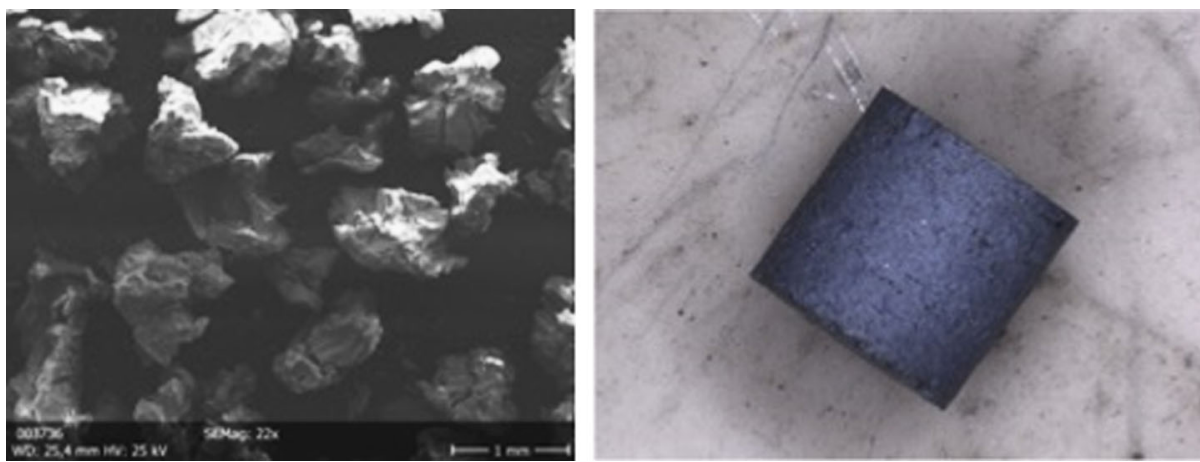

Fig. 5 (Left) Scanning electron micrograph of a sol-gel $\mathrm{Nd}_{2} \mathrm{O}_{3}$ material calcined at $650{ }^{\circ} \mathrm{C}$ in air. (Right) Pellet cold pressed and sintered. (The images above are reprinted from Watkinson et al. (2019); RightsLink Elsevier License 4623810907621) 
was conducted to identify if such a surrogate with a specified stoichiometry could be created and to understand the effect of the variables of the synthesis route on particle properties, with the objective of producing particles with a range of properties. The standard methods of oxalate co-precipitation and subsequent thermal decomposition (calcination) were used. Numerous analytical techniques were used to verify Ce-Nd oxide solid solution production and to characterise its composition. The effect of co-precipitation conditions (e.g. temperature and stirring rate) on particle morphology and size were found to be important, as well the influence of the calcination process on subsequent oxide particle properties (Watkinson et al. 2017a). Although this solid solution work is less relevant to the utilisation of $\mathrm{Am}_{2} \mathrm{O}_{3}$ for future ESA radioisotope power systems it is relevant to building a more comprehensive body of knowledge related to oxides of americium and their surrogates.

More recently, in collaboration with JRC, Watkinson et al. (2019) used the sol-gel method and oxalate-derived calcination to produce $\mathrm{Nd}_{2} \mathrm{O}_{3}$, and successfully sintered the powder produced via both these routes. Other work performed by JRC Karlsruhe focused on the production of uranium-stabilised americium oxide ceramic in order to maintain the cubic form of americium oxide over a large range of temperature and oxygen potential, including reductive sintering conditions. The produced ceramic had a measured specific power output of $0.082 \mathrm{~W}_{\text {th }} / \mathrm{g}$. This reduced specific power is due both to introduction of uranium in the oxide and the presence of a significant amount of ${ }^{237} \mathrm{~Np}$ (daughter element of ${ }^{241} \mathrm{Am}$ ). It has to be compared to the theoretical specific power of pure americium oxide of $0.101 \mathrm{~W}_{\mathrm{th}} / \mathrm{g}$ for $\mathrm{AmO}_{2}$ and $0.104 \mathrm{~W}_{\mathrm{th}} / \mathrm{g}$ for $\mathrm{Am}_{2} \mathrm{O}_{3}$. However, despite this drawback, uranium-stabilised americium oxide present several advantages on the material properties: stabilisation of the fluorite like structure which have excellent behaviour under alpha self-irradiation, pellet integrity preserved under occidental oxidizing condition improving material safety due to maintain of cubic structure, good behaviour during sintering, good host for americium decay product with the neptunium integrating the material structure.

For images of americium-based pellets and further details of the consolidation work carried out with americium oxides, the readers are referred to Sarsfield et al. $(2017,2018)$ and Vigier et al. (2018).

\section{Developing the Heat Source for RTGs and RHUs}

\subsection{Heat Source Architecture}

Once the fuel has been consolidated into pellets or discs, the fuel is then inserted into a multilayer containment system that is generally defined as the heat source. The containment approach is based on "defence in depth" with multiple layers of containment ensuring that risk of dispersal of the radioisotope is minimised under launch accident conditions. Containment directly linked to launch safety and radiological protection is described in detail by Barco et al. (2019a), Williams et al. (2013) and the ESA Safety Policy on the Use of Nuclear Power Sources (ESA 2019).

The schematic diagrams for the latest design for the heat source architectures for both the RHU and the RTG are shown in Fig. 6 below. The colour coding facilitates the distinction between the layers from the fuel to the metal cladding to the outer carbon-carbon composite aeroshell.

In the case of the RTG heat source design, the six-sided structure is designed specifically so that the majority of the surface area (i.e. larger sides and ends of the heat source) are insulated, when inside the RTG system, and the smaller sides are used as interfaces for the 

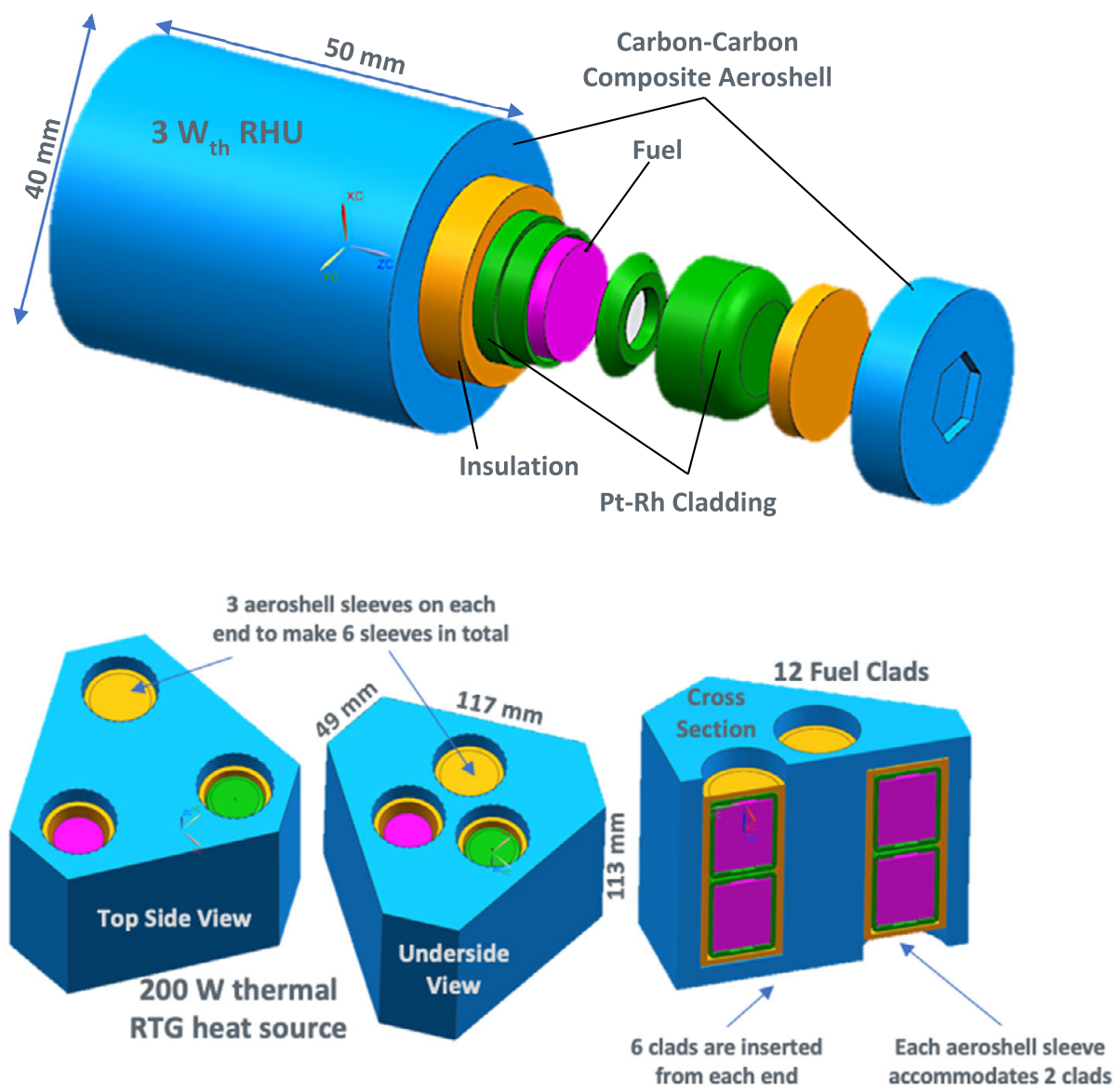

Fig. 6 (Top) Architecture for the RHU with colour coded elements: fuel (pink), platinum-rhodium cladding (green), insulation (orange), carbon-carbon composite aeroshell (blue). (Bottom) The latest design of the $200 \mathrm{~W}$ thermal heat source for the RTG system that incorporates 12 fuel clads. There are 6 aeroshell sleeves to accommodate the 12 clads, 3 on each side of the aeroshell. Each sleeve accommodates 2 clads

thermoelectric generators (see Sect. 4). In addition, the six-sided structure allows the fuel to occupy the smallest volume when compared to placing all the fuel on-axis. The volume occupied by the fuel affects the specific power of the RTG because it drives the volume of the whole system.

\subsection{Platinum Alloy Cladding}

The first containment layer in the heat source is a cladding structure made from a platinum $20 \%$-rhodium alloy. This welded cladding structure surrounds the fuel and forms a sealed source that can be inserted in the outer containment layer described in greater detail in Sect. 3.3.

Work carried out by the Oak Ridge National Laboratory and others in the 1960s and 1970s (Jaffee et al. 1961; Inouye et al. 1972) on the behaviour of candidate cladding materials and metals used in cermet fuels revealed that $\mathrm{Pt}, \mathrm{Rh}$ and $\mathrm{Pt}-\mathrm{Rh}$ alloys less likely to 
oxidise at the operating temperatures of americium-based nuclear power systems. The work outlines that this likelihood is 4 orders of magnitude less than iridium; 7 orders of magnitude less than tungsten, tantalum and niobium and 8 orders less than molybdenum and rhenium. These operating temperatures can range between $300{ }^{\circ} \mathrm{C}$ and $600{ }^{\circ} \mathrm{C}$ depending on whether the heat source is an RHU or an RTG heat source respectively.

Oxidation resistance is a driver for post-impact isotope containment capability given that changes to the structure of the cladding could compromise its mechanical properties; therefore, maximising this resistance is important in the design of the inner containment. A detailed investigation (led by the University of Leicester in collaboration with JRC, Karlsruhe) is currently underway to assess the compatibility of Pt-Rh alloys in contact with americiumbased oxide ceramics under a range of environments and will be the subject of future publications.

The experimental study by Inouye et al. (1972) highlighted that Pt alloys are oxidation resistant to at least $1200{ }^{\circ} \mathrm{C}$, with no measurable changes at this temperature after a 200 hour oxidation experiment when compared to Mo and Ta. The study also showed that the inclusion of $\mathrm{W}$ in Pt-Rh alloys improves strength and, contrary to the expected inverse relationship between strength and ductility, significantly increases ductility above $1100{ }^{\circ} \mathrm{C}$, a property that is unique to these alloys. The presence of $\mathrm{Hf}$ and $\mathrm{Ti}$ in the alloy can increase strength, increase recrystallisation temperature and reduce grain growth. Recrystallization tends to reduce strength and hardness while increased grain growth lowers yield stress. One key element in the report by Inouye et al. (1972) highlights that toughness yield strengths that can exceed $80 \%$ of ultimate tensile strengths. This is important when considering that the cladding could be subjected to a severe impact as a consequence of a launch accident (Barco et al. 2019a).

Both $\mathrm{Pt}$ and $\mathrm{Rh}$ produce binary eutectics in the presence of carbon at around the same temperature of $\sim 1738{ }^{\circ} \mathrm{C}$ (Yamada et al. 2001). This is relevant because a carbon-carbon composite aeroshell structure surrounds the fuel clads. However, Pt-30\% Rh maintains an elongation to failure greater than $30 \%$ even after exposure to graphite at $1700{ }^{\circ} \mathrm{C}$ (giving the potential to create C-Pt and C-Rh eutectics) (Tate 1982). This elongation to failure is also maintained down to ambient temperatures. The phase diagram for the americium-platinum system is described in detail by Schulz (1976) and shows that for Pt metal there is a eutectic formed with americium at $\sim 1580{ }^{\circ} \mathrm{C}$. Schulz also highlights that heating $\mathrm{Am}_{2} \mathrm{O}_{3}$ in a reducing atmosphere at $1200{ }^{\circ} \mathrm{C}$ with $\mathrm{Pt}$ for 40 hours will produce an intermetallic with $\mathrm{Pt}$ $\left(\mathrm{Pt}_{5} \mathrm{Am}\right)$; this is consistent with the increased vapour pressure of Am when working with $\mathrm{Am}_{2} \mathrm{O}_{3}$ as opposed to $\mathrm{AmO}_{2}$ (Sarsfield et al. 2018). The work by Schulz (1976) shows that there is an intermetallic between $\mathrm{Rh}$ and $\mathrm{Am}$, which forms when heating $\mathrm{AmO}_{2}$ with $\mathrm{Rh}$ in a reducing atmosphere at $1550{ }^{\circ} \mathrm{C}$ for 60 hours.

The referenced studies outline some of the key critical temperatures that could affect the properties of the cladding and lead to important safety considerations. These temperatures are being used as design drivers in the architectures of the heat sources for both the RHU and RTG being developed for ESA.

The most technically mature approach for americium-fuelled RTGs and RHUs, in terms of total number of systems launched and test data available, is to use a Pt-based alloy as a cladding layer around the fuel pellet. Pt-Rh alloys are most compatible, stable and least reactive materials that could meet the safety requirements that have been defined as part of the ESA programme. These alloys offer a good starting point for developing and testing a primary containment layer for americium systems.

The oxygen released by the americium-oxide fuel at elevated temperature, or the buildup of helium over time as a consequence of alpha decay, could pressurize the clad from 


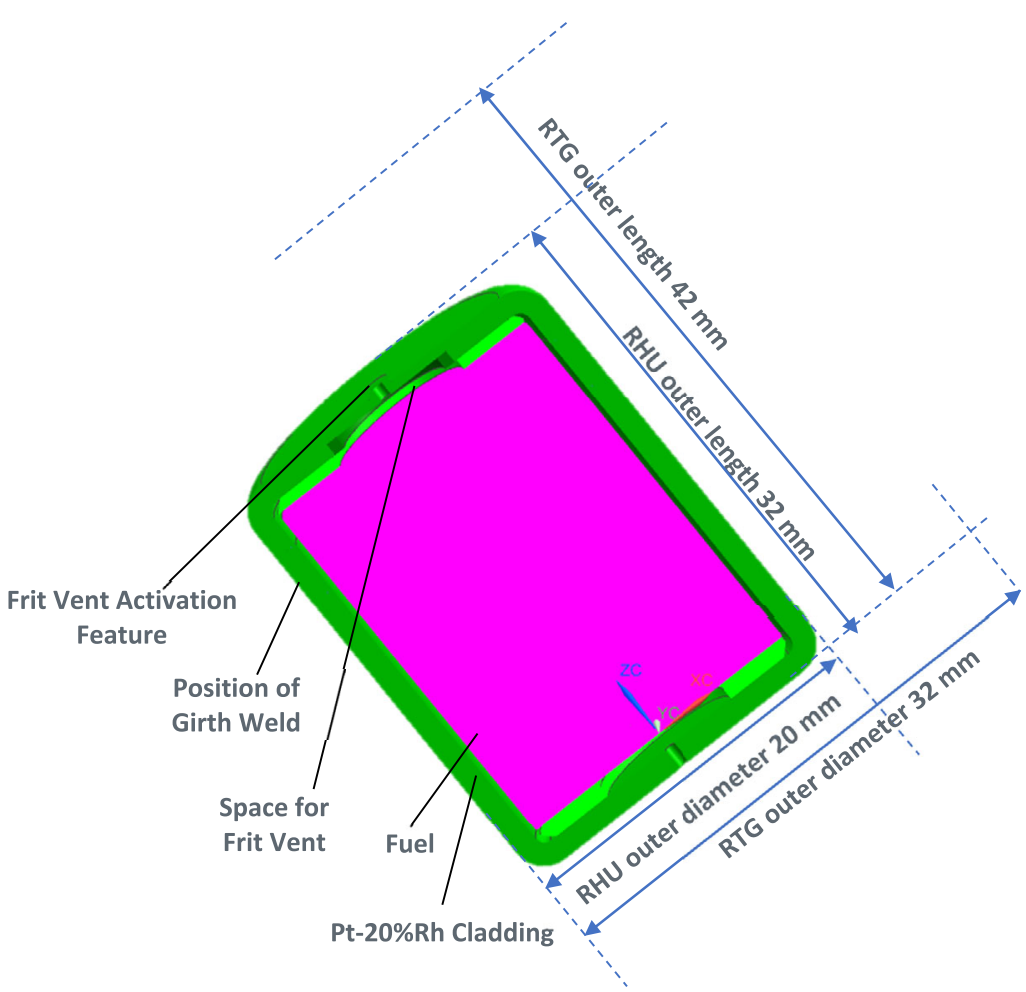

Fig. 7 General architecture of the RHU and RTG clads. One key feature is that the RTG clad is a scaled-up version of the RHU clad which shares the frit vent design of the RHU clad

within. To avoid this pressurization, a vent hole covered by a porous frit is required in the cladding design. This allows the gases to be released and reduces the risk of the clad breaching (Johnson 1984). Frit vents (made of sintered pure platinum powder) for European RTG systems are currently under development using proprietary methods being developed by the University of Leicester and will be reported in the literature when results become available. To the best knowledge of the authors, platinum frit vents are not commercially available in Europe.

The general architecture for the platinum cladding structures for the RTG and RHU heat sources is provided in Fig. 7. The only differences between the RTG clad and RHU clad is that the RTG clad has a larger diameter ( $32 \mathrm{~mm}$ outer diameter is as opposed to $20 \mathrm{~mm}$ for the RHU) and length (42 $\mathrm{mm}$ as opposed to $32 \mathrm{~mm}$ for the RHU). Both are made from same alloy (Pt-20Rh), have the same wall thickness (approximately $2 \mathrm{~mm}$ ), are machined from a solid bar to produce the net shapes, have a threaded girth seal which can be welded, and both share the same frit vent design.

\subsection{Carbon-Carbon Composite Aeroshell}

Surrounding the cladding are two layers: an insulating layer and a carbon-carbon composite aeroshell. This approach is a standard way of protecting the cladding and fuel from accident conditions and is reflected in the US GPHS and light-weight radioisotope heater unit (LWRHU) designs (Hula 2015). 
The layer immediately surrounding the primary containment or cladding is an insulation layer. A compliant insulating graphitic foam is the latest baseline design. This insulation layer prevents the cladding and fuel from exceeding specific temperatures defined in the first iteration of the safety requirements for a European RTG and RHU when these systems are exposed to extreme external temperatures (see Sect. 3.3.1 and 3.4). These extreme nonoperational conditions can be experienced under different accident scenarios e.g. Earth reentry, launch-pad fires. This effect has been shown in a study by El Genk and Tournier for the US LWRHU (El Genk and Tournier 2001).

Surrounding the cladding and the fuel is the protective ablative aeroshell material that provides a thermo-mechanical interface between the heat source and the thermoelectric converters in the case of the RTG and an interface with the thermal management system of the spacecraft in the case of the RHU. A number of 2.5D and 3D carbon-carbon composites were identified as options for the European RTG and RHU heat sources. These composites are available as commercial off-the-shelf products produced for aerospace applications in France. Three-dimensional carbon-carbon composites are produced by Ariane Group and grouped under the SEPCARB ${ }^{\circledR}$ (Lacoste et al. 2002) registered product name. The SEPCARB $^{\circledR}$ family of composites are the baseline for the ESA RTG and RHU heat source aeroshells. Both 2.5D and 3D grades of materials produced by Mersen in France listed as A035 and AM252 (Mersen 2019) have been evaluated.

Thermal and mechanical modelling of the heat sources has been carried out by Barco et al. (2019b). The performance of the aeroshell materials under Earth re-entry accident conditions has been simulated and are summarised below.

\subsubsection{Earth Re-Entry Heating Simulation of Aeroshell Materials}

In 1957, Allen and Eggers (1957) studied the motion and heating of ballistic missiles entering the Earth's atmosphere at supersonic speeds. From their study, they derived a multitude of analytical equations, which could be applied to a broad range of re-entry scenarios. Following this work, a number of authors developed simplified expression for stagnation point aerothermal heating including: Fay and Riddell (1958), Detra and Hidalgo (1961) and, more recently, Brandis and Johnston (2014). Putnam and Braun (2015) revisited the Allen and Eggers analytical solutions and produced an extended and enhanced version. This extended and enhanced version can be applied to atmospheric re-entry scenarios for RTGs and RHUs; allowing the velocity, $V$, the acceleration magnitude, $a$, and the convective heat flux, $Q$, to be calculated at the stagnation point for any stage in the descent.

These models provide an approximation of the re-entry process. A more complete picture can be developed by modelling which incorporates explicit treatments for rarefied, laminar and turbulent regimes. These more sophisticated models included drag coefficients that were based on collective predictions using appropriate aerodynamic prediction tools based on works such as Hoerner (1965) and Klett (1964). In all cases tumbling was not assumed or modelled in detail. Both convective and radiative heat fluxes are considered by these more complete models. In order to develop models for a number of re-entry cases, the following RTG and RHU ballistic properties were calculated and used in the simulations (Table 1). In addition, a number of discrete cases were defined for the re-entry simulations. Two of these are provided in Table 2 .

Using the Allen and Eggers (1957) and enhanced Putnam and Braun (2015) methodologies, the results shown in Table 3 were obtained for the RTG and RHU. In Table 4, the results of the more sophisticated model that considers the most important re-entry effects (as described above) are shown. In addition, the Fluid Gravity Engineering Ablation Code 
Table 1 RTG and RHU ballistic properties for re-entry simulations

${ }^{\mathrm{a}}$ The RTG re-entering on short side

${ }^{b}$ The RHU re-entering on end face or on the flat

Table 2 RTG and RHU re-entry scenarios

\begin{tabular}{|c|c|c|}
\hline Property & RTG side on ${ }^{\mathrm{a}}$ & RHU end on ${ }^{b}$ \\
\hline Mass (kg) & 6.1 & 0.17 \\
\hline Reference Area $\left(\mathrm{m}^{2}\right)$ & 0.019 & 0.0014 \\
\hline $\begin{array}{l}\text { Ballistic Coefficient }\left(\mathrm{kg} \mathrm{m}^{-2} \text { ) }\right. \\
\text { (indicative of high speeds) }\end{array}$ & 337 & 66 \\
\hline $\begin{array}{l}\text { Ballistic Coefficient }\left(\mathrm{kg} \mathrm{m}^{-2}\right) \\
\text { (indicative of subsonic speeds) }\end{array}$ & 598 & 133 \\
\hline
\end{tabular}

\begin{tabular}{lcc}
\hline Parameter & $\begin{array}{l}\text { Comet return } \\
(\mathrm{CR})\end{array}$ & $\begin{array}{l}\text { Shallow re-entry } \\
(\mathrm{SE})\end{array}$ \\
\hline Initial Velocity $\left(\mathrm{ms}^{-1}\right)$ & 12106 & 11000 \\
Flight Path Angle (degrees) & -11 & -6.5 \\
Altitude $(\mathrm{m})$ & 78000 & 125000 \\
\hline
\end{tabular}

Table 3 Analytical results for the RTG and RHU

\begin{tabular}{lcccrr}
\hline Parameter & \multicolumn{2}{l}{ Comet return $(\mathrm{CR})$} & & \multicolumn{2}{c}{ Shallow re-entry (SE) } \\
\cline { 2 - 3 } & RTG & RHU & & RTG & RHU \\
\hline Peak Convective Heat Flux $\left(\mathrm{MW} \mathrm{m}^{-2}\right)$ & 36 & 29 & 21 & 17 \\
Altitude at Peak Convective Heat Flux $(\mathrm{km})$ & 43 & 57 & 48 & 61 \\
Total Heat Load $\left(\mathrm{MJ} \mathrm{m}^{-2}\right)$ & 576 & 285 & 716 & 474 \\
\hline
\end{tabular}

Table 4 Detailed modelling results for the RTG, which includes a prediction of the recession depth of the aeroshell material

\begin{tabular}{|c|c|c|c|c|}
\hline \multirow[t]{2}{*}{ Parameter } & \multicolumn{2}{|c|}{ Comet return $(\mathrm{CR})$} & \multicolumn{2}{|c|}{ Shallow re-entry (SE) } \\
\hline & RTG & RHU & RTG & RHU \\
\hline Peak Convective Heat Flux $\left(\mathrm{MW} \mathrm{m}^{-2}\right)$ & 37 & 31 & 12 & 14 \\
\hline Altitude at Peak Convective Heat Flux (km) & 39 & 57 & 56 & 66 \\
\hline Total Heat Load $\left(\mathrm{MJ} \mathrm{m}^{-2}\right)$ & 628 & 448 & 811 & 654 \\
\hline Predicted Recession (mm) & 4.6 & 2.3 & 4.9 & 3.3 \\
\hline Ground Impact Vel. $\left(\mathrm{ms}^{-1}\right)$ & 97 & 46 & 97 & 46 \\
\hline
\end{tabular}

(FABL) was used to determine the recession depth for the RTG and RHU re-entering on preferred orientation and also provided ground impact velocities.

As shown in Table 5, the simulations have also tested the thermo-mechanical stresses, minimum reserve factors and aeroshell surface and predicted fuel temperatures under different re-entry scenarios. In addition, peak external aeroshell temperatures above $3500{ }^{\circ} \mathrm{C}$ were obtained but for very short periods of time of order 6 seconds with the temperature dropping to $2500{ }^{\circ} \mathrm{C}$ around $10 \mathrm{~s}$ after reaching the peak temperature. These simulations do not assume any tumbling and represent a worst case. 
Table 5 RTG and RHU ballistic properties for re-entry simulations

\begin{tabular}{|c|c|c|c|c|}
\hline \multirow[t]{2}{*}{ Property } & \multicolumn{2}{|c|}{ RTG side on ${ }^{a}$} & \multicolumn{2}{|c|}{ RHU end on ${ }^{b}$} \\
\hline & CR & SE & $\mathrm{CR}$ & SE \\
\hline Maximum Compressive Strength (MPa) & 99 & 80 & 96 & 106 \\
\hline Minimum Reserve Factor & 1.2 & 1.5 & 1.2 & 1.1 \\
\hline Surface Temperature $\left({ }^{\circ} \mathrm{C}\right)$ & 2700 & 2600 & 2800 & 2500 \\
\hline (time $t$ in $\mathrm{s}$ ) & $(13)$ & $(110)$ & (7) & $(51)$ \\
\hline Peak Surface Temperature $\left({ }^{\circ} \mathrm{C}\right)$ & 3531 & 3080 & 3159 & 2912 \\
\hline (time $t$ in $\mathrm{s}$ ) & $(18)$ & $(84)$ & $(11)$ & $(66)$ \\
\hline Max Core Fuel Temperature $\left({ }^{\circ} \mathrm{C}\right)$ & $700^{c}$ & $900^{\mathrm{c}}$ & $700^{\mathrm{d}}$ & $700^{\mathrm{d}}$ \\
\hline
\end{tabular}

${ }^{\mathrm{a}}$ The RTG re-entering on short side

${ }^{\mathrm{b}}$ The RHU re-entering on end face or on the flat

${ }^{\mathrm{c}}$ The steady state temperature for the fuel is assumed to be between $650{ }^{\circ} \mathrm{C}$ and $680{ }^{\circ} \mathrm{C}$

${ }^{d}$ The steady state temperature for the fuel is assumed to be between $100{ }^{\circ} \mathrm{C}$ and $150{ }^{\circ} \mathrm{C}$, which is an underestimate and could be as high as $300^{\circ} \mathrm{C}$. This implies that the max core fuel temperature in the model could be an underestimate but still below $1000{ }^{\circ} \mathrm{C}$

Although some experimental evaluation of aeroshell materials has been carried out and some results are reported in the literature by Ambrosi et al. (2018) more testing is planned in 2019 and this will be reported in 2020. The experimental work carried out corroborates the modelling conducted to date. Further work on the impact of tumbling is underway.

\subsection{Summary of Heat Source Characteristics \& Critical Temperatures}

A summary of the heat source characteristics and fuel type in addition to the critical temperatures associated with operational and non-operational conditions are shown in Table 6 and Fig. 8 respectively. Figure 8 also includes parameters associated with the US LWRHU for comparison.

\section{Thermoelectric Converters for RTGs}

A number of generations and variations of bismuth telluride based thermoelectric converters or generators (TEGs) have been produced for the RTG system (Mesalam et al. 2018, 2019; Williams et al. 2014). Americium-based nuclear power systems have lower power densities and occupy larger volumes for equivalent power outputs than Pu-based systems and offer the option of targeting lower operational temperatures (Ambrosi et al. 2019; Barco et al. 2019a). Bismuth telluride TEGs that operate between $220{ }^{\circ} \mathrm{C}$ and room temperature are well-suited to these systems (Rowe 2006).

The thermoelectric generator production programme has focused on establishing the complete end-to-end capability in the UK, from the constituent elements, using appropriate materials processing and consolidation methods, through to segmentation into unicouples and module production. This approach is summarised in Fig. 9. Several generations of modules have been produced; however, the current baseline is a $40 \mathrm{~mm} \times 40 \mathrm{~mm}$ unit which has 161 couples in a $1.2 \mathrm{~mm} \times 1.2 \mathrm{~mm}$ cross section and $6 \mathrm{~mm}$ in height. These devices were optimised for this specific programme. Commercial standard production methods were used 
Table 6 Summary of the heat source characteristics

\begin{tabular}{|c|c|c|}
\hline System & ESA RTG & ESA RHU \\
\hline Fuel & ${ }^{241} \mathrm{Am}_{2} \mathrm{O}_{3}{ }^{\mathrm{a}}$ & ${ }^{241} \mathrm{Am}_{2} \mathrm{O}_{3}{ }^{\mathrm{a}}$ \\
\hline Total thermal power output $\left(\mathrm{W}_{\mathrm{th}}\right)$ & 200 from $2 \mathrm{~kg}$ of fuel ${ }^{\mathrm{b}}$ & 3 from $30 \mathrm{~g}$ of fuel ${ }^{\mathrm{b}}$ \\
\hline Thermal power output per fuel clad $\left(\mathrm{W}_{\mathrm{th}}\right)$ & $16.7^{\mathrm{b}}$ & $3^{b}$ \\
\hline Electrical power output $\left(\mathrm{W}_{\mathrm{e}}\right)$ & 10 (scalable to 50 ) & N/A \\
\hline Fuel Density ${ }^{c}$ & $85 \%$ to $95 \%$ & $85 \%$ to $95 \%$ \\
\hline Fuel Diameter (mm) & 27 & 15 \\
\hline Fuel Height $(\mathrm{mm})^{\mathrm{d}}$ & 35 & 23 \\
\hline Positive Margin on Fuel Volume $\mathrm{e}^{\mathrm{e}}$ & $9 \%$ & $16 \%$ \\
\hline Heat Source Mass (kg) & $6.5 \mathrm{~kg}$ to $6.75 \mathrm{~kg}^{\mathrm{e}}$ & $202 \mathrm{~g}$ \\
\hline Power System Mass & $10.8 \mathrm{~kg}$ & N/A \\
\hline \multirow[t]{2}{*}{ Containment architecture } & $\begin{array}{l}\mathrm{Pt}-20 \% \mathrm{Rh} \text { inner } \\
\text { containment }\end{array}$ & Pt-20\%Rh inner containment \\
\hline & $\begin{array}{l}\text { Carbon-Bonded } \\
\text { Carbon Fibre (CBCF) } \\
\text { insulation sleeve (or } \\
\text { equivalent) }\end{array}$ & $\begin{array}{l}\text { Carbon-Bonded Carbon } \\
\text { Fibre (CBCF) insulation } \\
\text { sleeve (or equivalent) }\end{array}$ \\
\hline Specific Electrical Power $\left(\mathrm{W}_{\mathrm{e}} / \mathrm{kg}\right)$ & $1\left(\right.$ at $\left.10 \mathrm{~W}_{\mathrm{e}}\right)$ & N/A \\
\hline Specific Thermal Power $\left(\mathrm{W}_{\mathrm{th}} / \mathrm{kg}\right)$ & 20 & 14.9 \\
\hline
\end{tabular}

\footnotetext{
${ }^{\mathrm{a}}$ This assumes a pure Am ceramic with no U content

${ }^{b}$ Based on approximately $0.1 \mathrm{~W}_{\text {th }}$ per gram of $\mathrm{Am}_{2} \mathrm{O}_{3}$. The uranium-stabilised americium oxide (Vigier et al. 2018) would result in approximately $0.08 \mathrm{~W}_{\text {th }}$ per gram of fuel material

${ }^{\mathrm{c}}$ At present the density of the consolidated fuel is likely to be within the range outlined

${ }^{\mathrm{d}}$ This is the total fuel height within the clad

${ }^{\mathrm{e}}$ This mass depends on the density of the sintered fuel
}

to maximise repeatability and success rates. Work done by Williams et al. (2014), for example, on modifying the thermoelectric materials by the addition of boron carbide, has resulted in improvements in mechanical properties without negatively impacting on thermoelectric properties.

An impedance spectroscopy technique that has been developed at Leicester by Mesalam et al. (2018) for the rapid evaluation of modules has been reported in detail in Mesalam et al. $(2018,2019)$. This method enables rapid diagnosis and isolation of material or manufacturing effects on module properties and performance.

As part of a collaboration that has been established with the University of Dayton, Ohio, USA, a number of radiation susceptibility and lifetime testing experiments have been carried out on the modules produced as part of the ESA programme.

A set of thermoelectric generators were exposed to a nominal $5 \times 10^{13}$ neutrons $/ \mathrm{cm}^{2}$ using a hard neutron spectrum (i.e. above $1 \mathrm{MeV}$ with $\mathrm{Cd}$ shielding absorbing thermal flux). The exposure was nominally equivalent to a 12-year Mars mission cycle (including a 1-year pre-launch assembly and storage, a 1-year cruise and 10-year surface mission) with a single $50 \mathrm{~W}$ electric americium-based RTG. The irradiation experiment was carried out at the Ohio State University reactor (see Fig. 10). The thermoelectric generators tested were unaffected by the neutron irradiation, as reported by Mesalam et al. (2019). 


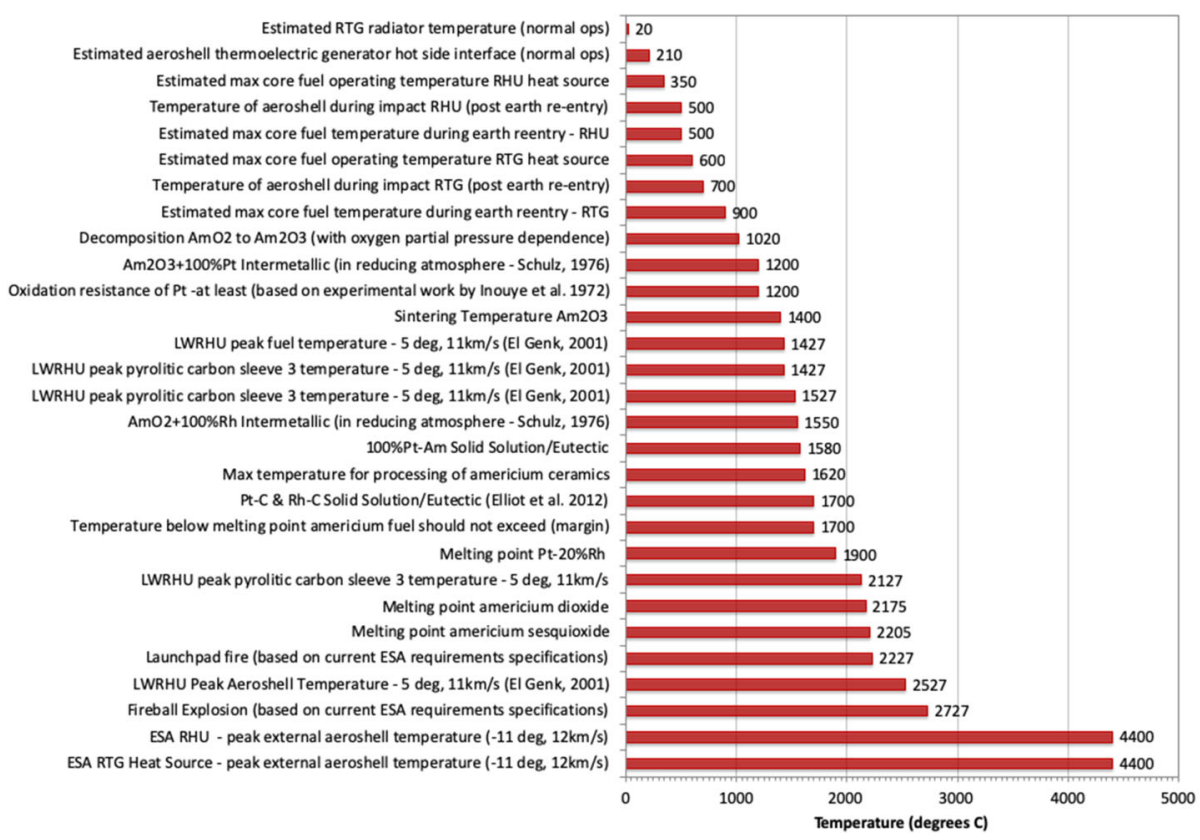

Fig. 8 Critical temperatures to consider in RTG and RHU designs
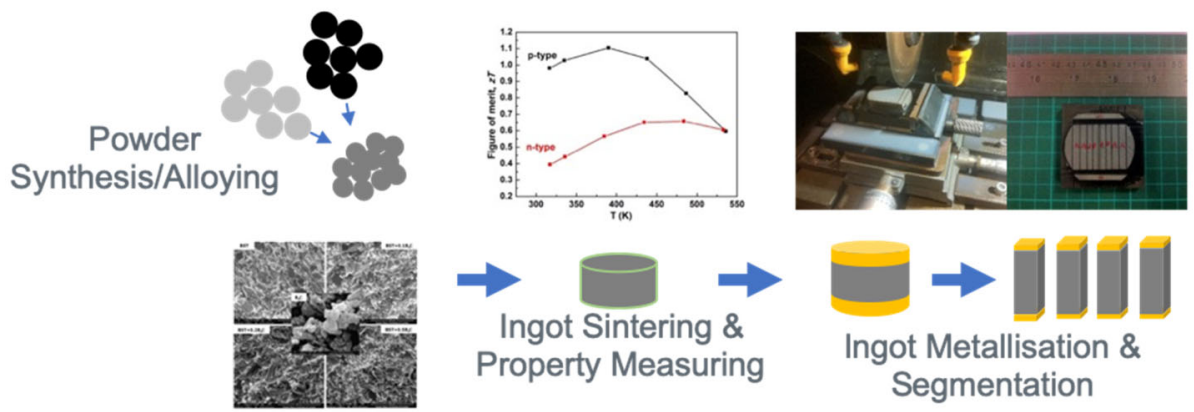

\section{Bismuth Telluride Compounds \& Composites}
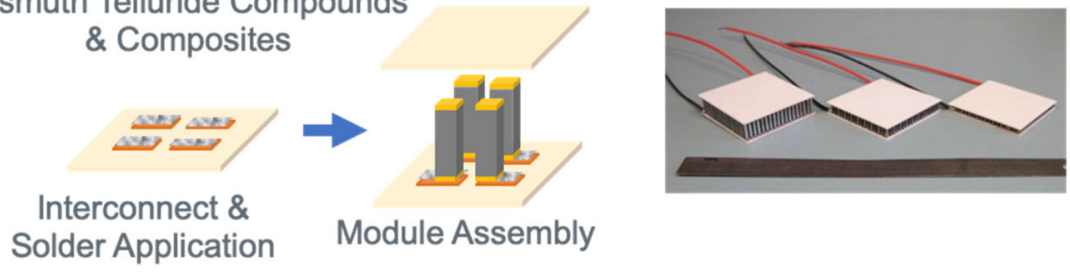

\section{Solder Application \\ Module Assembly}

Fig. 9 Component powders (bismuth, tellurium, selenium, antimony) are mixed in required stoichiometric ratios, milled and sintered to produce pucks (approximately $30 \mathrm{~mm}$ in diameter and $7 \mathrm{~mm}$ to $9 \mathrm{~mm}$ thick) of p-type and n-type semiconductor materials. The properties of the pucks are measured. Metallisation is via nickel plating step and each puck is subsequently segmented. Ceramic (alumina) substrates with copper electrical connections are prepared with solder tabs and modules are assembled to produce $40 \times 40 \mathrm{~mm}^{2}$ units each with 161 couples and with variable height ranging between $2 \mathrm{~mm}, 4 \mathrm{~mm}, 6 \mathrm{~mm}$ and $8 \mathrm{~mm}$ (all have been demonstrated) 

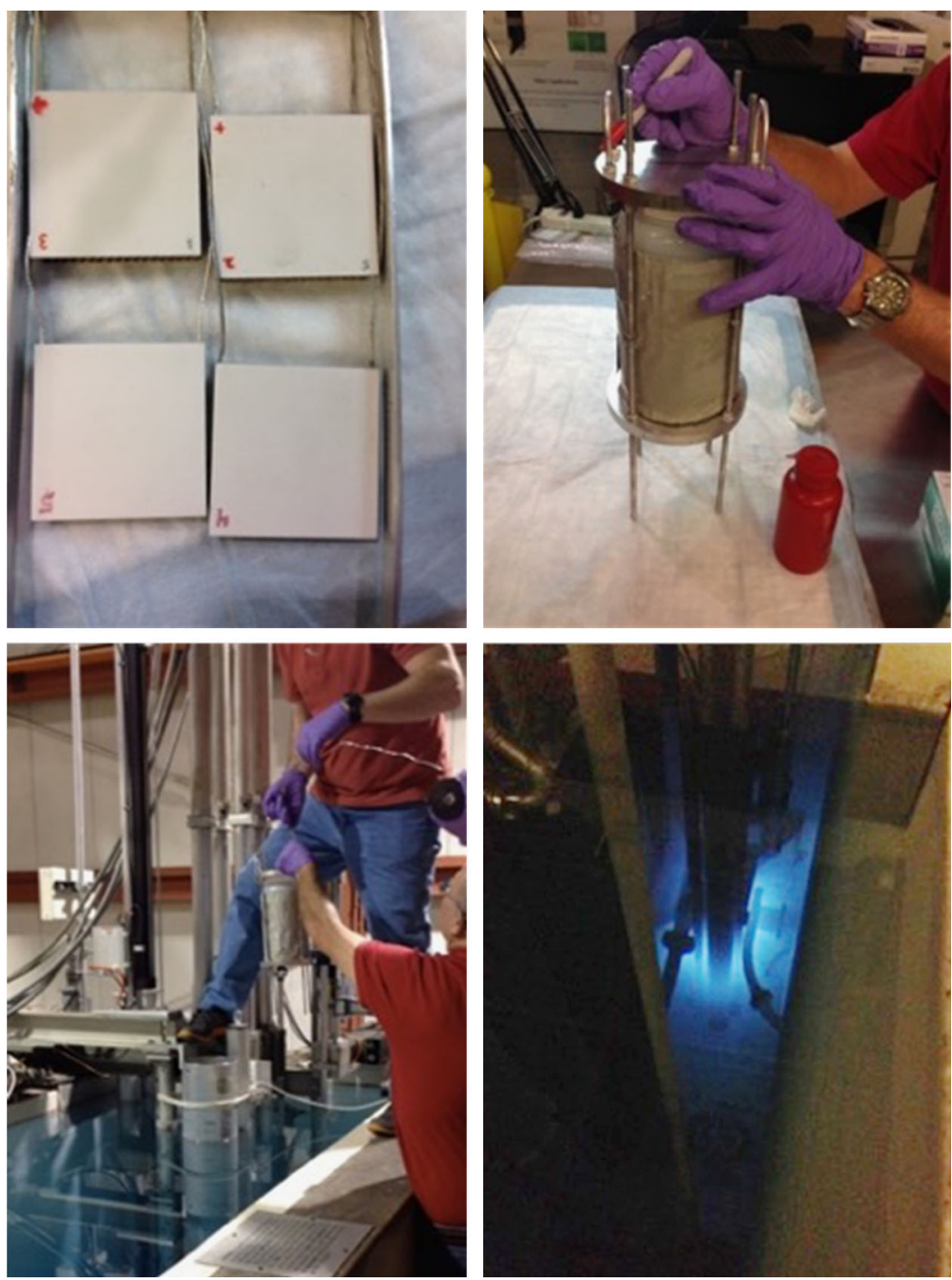

Fig. 10 Neutron irradiation of thermoelectric generators, showing the 4 TEGs (top left) in a watertight container shielded with $\mathrm{Cd}$ (top right) to absorb thermal neutrons is lowered into the reactor pool (bottom images). (The images above are reprinted from Mesalam et al. (2019) under Creative Commons Attribution (CC BY) license http://creativecommons.org/licenses/by/4.0/)

Two thermoelectric generators have been tested under vacuum for a total of 10,000 hours. One of the TEGs was used as a control and was simply placed in the vacuum environment. The second unit was connected to an external load and was exposed to hot side temperatures of around $150{ }^{\circ} \mathrm{C}$ and cold side temperatures of around $20^{\circ} \mathrm{C}$. Temperature values were increased more recently to $160{ }^{\circ} \mathrm{C}$. Thermoelectric efficiency can be determined using a dimensionless figure-of-merit as shown in Eq. (1).

$$
Z T=\frac{S^{2} T}{\rho \lambda}
$$


In Eq. (1), $T$ is temperature, $S$ is the Seebeck coefficient, $\rho$ is the electrical resistivity and $\lambda$ is the thermal conductivity. The data in Table 7 include values for these thermoelectric device characteristics. Taking Eq. (1) into account, the results in Table 7 show no discernible changes after 10,000 hours of operation. This is aside from a small change in electrical resistance due to $5{ }^{\circ} \mathrm{C}$ changes in ambient thermal environment in which the impedance spectroscopy measurements were carried out. This result is consistent with the literature and the degradation of bismuth telluride thermoelectric generators is averted or minimised by maintaining the maximum operational hot side temperature below $220{ }^{\circ} \mathrm{C}$. More information can be found in Mesalam et al. (2019).

The work done by Mesalam et al. (2019) and the lifetime testing carried out to date suggest that the lower operating temperature and use of bismuth telluride make this RTG system design relatively unsusceptible to changes over time. The expected or predicted degradation of the thermoelectric devices is at the $<1 \%$ per year level. This implies that the end-of-life power output of a $10 \mathrm{~W}$ RTG for a 10-year mission would be at least $9 \mathrm{~W}$. Further extended testing is planned as the programme migrates to flight model development of both the thermoelectric generators and power systems. In addition, the most recent design of the RTG system that has been developed (see Sect. 5.4) facilitates the replacement of the thermoelectric generators should flight delays and pre-launch storage periods impact on the beginning-of-mission performance of the system.

\section{The Evolution of the RTG System Architectures}

The architecture of the RTG system has evolved since the programme began in 2010. Three generations of RTG flight design have been produced, each building and improving on previous designs. Three prototype devices reflecting the flight designs have been built and tested. The current technology readiness level (TRL) of the RTG system is 4 (i.e. breadboard functional verification in laboratory environment). The University of Leicester team is currently working on a further iteration of the flight design and is in the process of building a flight system to TRL 5 (i.e. breadboard critical function verification in relevant environment) on the ESA ISO scale (ECSS 2019). The plan is for the new system to be completed in 2021. A roadmap to develop a flight ready RTG at TRL8 by 2028 has been developed in collaboration with ESA, which would be compatible with an outer solar system mission target of around 2030.

\subsection{Early RTG Flight System Designs}

Early flight designs assumed a basic building block of a $10 \mathrm{~W}$ electric scalable design that could be scaled to produce $50 \mathrm{~W}$ of electrical power. The following sections briefly summarise these initial concepts. The on-axis fuel configuration in early designs reflected a configuration similar to the US SNAP 19 heat source design (Hula 2015). The early designs assumed the fuel in each $10 \mathrm{~W}$ unit would be a stack of two $\mathrm{Am}_{2} \mathrm{O}_{3} 40 \mathrm{~mm}$ diameter and $56 \mathrm{~mm}$ thick pellets clad in an iridium alloy. The hypothesis was that the system would be able to achieve a $6 \%$ conversion efficiency from $175 \mathrm{~W}$ of thermal power output from the fuel. This configuration is shown in Fig. 11.

The thermoelectric generators were assumed to be lead telluride based and in contact with a polygonal aeroshell structure. The lead telluride thermoelectric generators had a unicouple height ranging between 8 and $15 \mathrm{~mm}$ with $2.8 \times 2.8 \mathrm{~mm}^{2}$ in cross sectional area. The optimum operational range for the thermoelectric generators was determined as follows: hot 


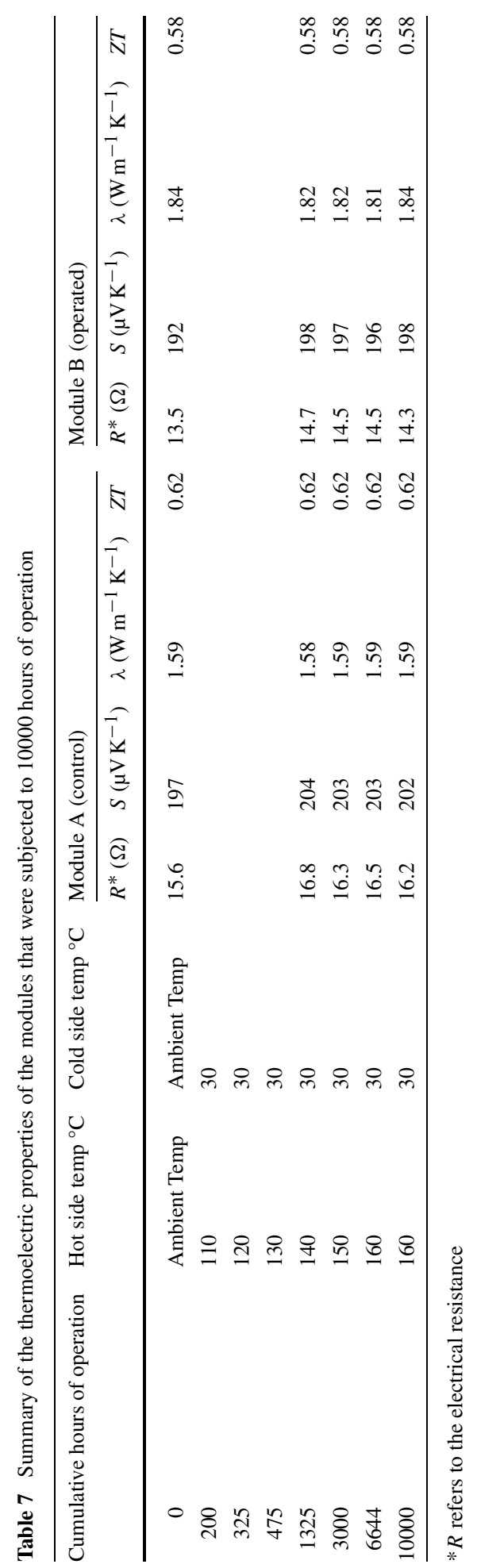




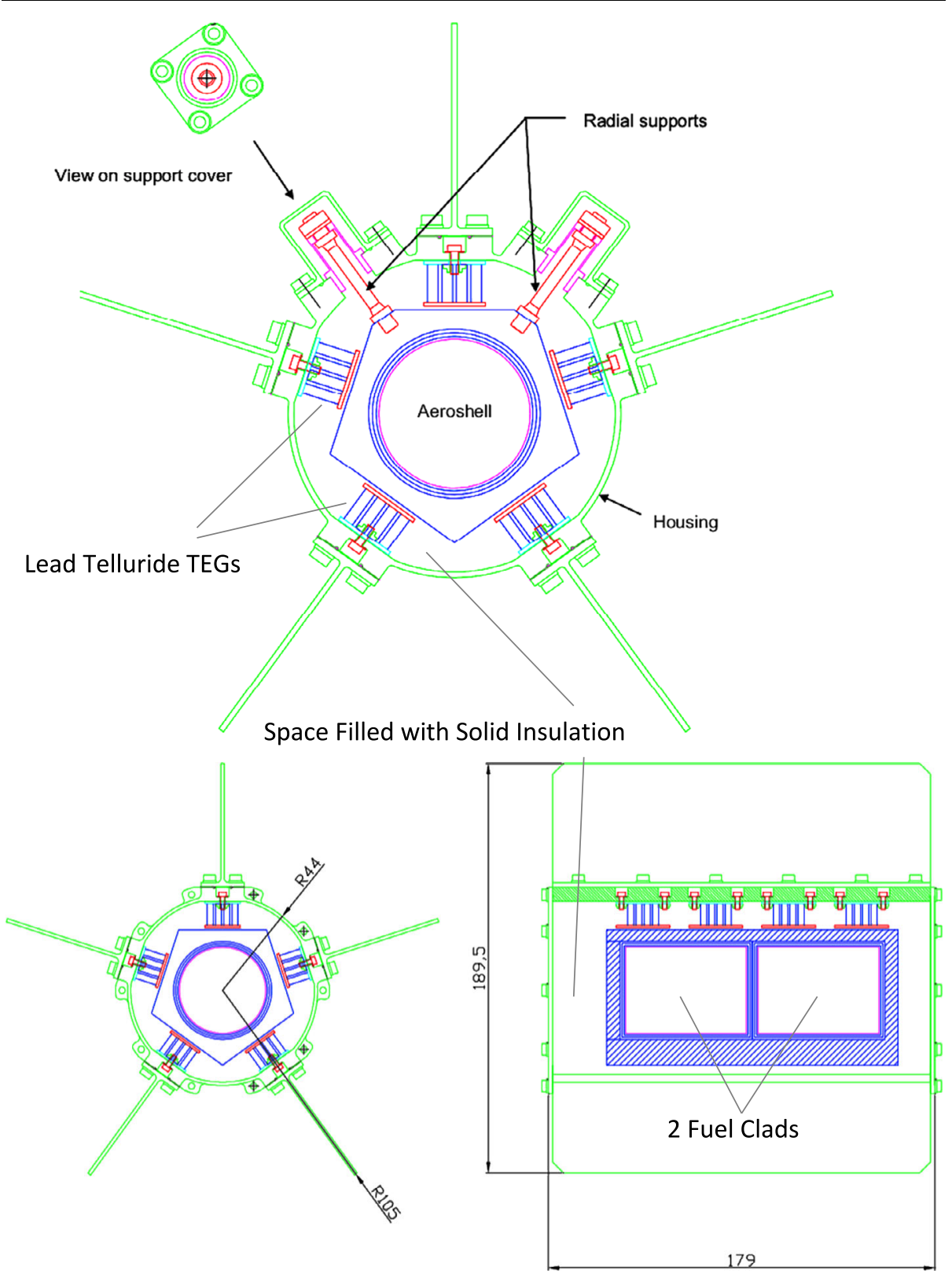

Fig. 11 The first RTG flight design which shows the fuel on axis and TEGs surround the polygonal aeroshell. The dimensions shown are in $\mathrm{mm}$

side operating temperature was $530^{\circ} \mathrm{C}$ and cold side of $175^{\circ} \mathrm{C}$ set by the aluminium 2219 (T852) radiator.

Alternative radiator material choices included AM162 (AlBeMet); an alloy consisting of $62 \%$ aluminium and $38 \%$ beryllium. AlBeMet is a material that retains a significant proportion of the high stiffness-to-weight and high thermal conductivity of pure beryllium with 
an improvement in mechanical strength. Room temperature strength of AM162 AlBeMet is comparable to 2219 aluminium alloy (375 MPa versus $372 \mathrm{MPa}$ respectively), but a higher fraction of this is retained at $200{ }^{\circ} \mathrm{C}$ i.e. $76 \%$ of ultimate tensile strength and $81 \%$ of yield strength. For 2219 alloy both of these values reduce to $50 \%$. At $200{ }^{\circ} \mathrm{C}$ AlBeMet offers significantly superior performance to 2219 aluminium alloy with a modulus to density ratio that is about four times higher, strength to density ratio that is about two times higher and thermal conductivity to density ratio that is about two times higher. For these reasons AlBeMet was considered an option for weight savings, specific power considerations and improvement in overall mechanical performance. However, the presence of beryllium made it more challenging from a handling and procurement perspective and therefore this was not considered as an option at the time.

These early designs had calculated specific power values of around $2.1 \mathrm{~W} / \mathrm{kg}$ for $10 \mathrm{~W}$ electric units.

The transition from design to practical testing in the lab outlined that the European design, based on americium fuel, would operate at lower temperatures than predicted and that the system level efficiency target of 6\% was too high. This is discussed in detail in Sect. 5.2. One additional concern was the utilisation of lead telluride based thermoelectric generators operating above the critical sublimation temperature of tellurium as described in a study by Okamoto et al. (1990) which includes the phase diagram for bismuth telluride alloys.

The implication of lower operating temperatures offered a number of benefits:

- A transition to bismuth telluride thermoelectric generators with well-established industrial production methods. These generators are currently the baseline. (See Sect. 4.)

- Operational temperatures below the critical sublimation temperatures of tellurium (Okamoto et al. 1990).

- Longer operational lifetimes with reduced degradation of TEGs (Mesalam et al. 2019).

- Use of off-the-shelf standard materials for all components due to benign operating temperatures.

- Transition from iridium to platinum alloys for cladding. (See Sect. 3.2.)

Scaling the overall power output of these early design to $50 \mathrm{~W}$ electric units presented some additional challenges. With the fuel on axis two options were explored.

- The first was stacking $10 \mathrm{~W}$ electric units to produce a $50 \mathrm{~W}$ electric unit. In this configuration the modularity was at system level and the overall length of the RTG expanded from $179 \mathrm{~mm}$ to $895 \mathrm{~mm}$ and the specific power decreased from $2.1 \mathrm{~W} / \mathrm{kg}$ to $1.3 \mathrm{~W} / \mathrm{kg}$. In this configuration the radiator fins were segmented or slotted.

- The second was to make the heat source the modular element and design an optimised $50 \mathrm{~W}$ electric unit. This resulted in an increase in overall length from $179 \mathrm{~mm}$ to $669 \mathrm{~mm}$ and an increase in diameter from 190 to $277 \mathrm{~mm}$ due to the fins being part of the primary structure. The specific power dropped from $2.1 \mathrm{~W} / \mathrm{kg}$ to $1.9 \mathrm{~W} / \mathrm{kg}$.

These designs incorporated a $20 \%$ margin and the specific power reflects the inclusion of the margin. The design assumed lead telluride operating temperatures and did not factor in any additional requirements to increase radiator area caused by a switch to bismuth telluride TEGs and lower cold side operating temperatures i.e. $20^{\circ} \mathrm{C}$ as opposed to $175^{\circ} \mathrm{C}$.

\subsection{Building the First Small-Scale RTG Laboratory Prototype}

The design of the first small-scale breadboard RTG was based on the flight system architecture developed in the early phases of the project as shown in Sect. 5.1. The principle of a clad 
fuel element on-axis forming a compact heat source surrounded by a polygonal aeroshell to reduce the overall system volume and mass was a core element of the design philosophy. The prototyping process retained almost all of the features of the design described in Sect. 5.1 with modifications made either due to availability of specific materials or to facilitate the assembly process. Assembly integration and testing, operation in a vacuum chamber and integration of the system with the control electronics and software were successfully completed. Figure 12 shows the configuration of the breadboard and the assembled system in a vacuum chamber. Key design features include:

- A multilayer gold-plated heat shield, which effectively acts as a stiff MLI-like structure and ensures that the heat flows from the heat source through the thermoelectric generators and minimises losses.

- The ability to test up to 4 thermoelectric generators mounted on specially designed blocks that incorporate springs to compressively couple the thermoelectric generators to the heat source.

- Inconel support struts designed to minimise heat losses.

- The ability to operate in vacuum and with a cover gas.

- Evolution in thermal management system from liquid nitrogen-based system with copper heat pipes to a pumped fluid loop with cooling plates (Fig. 13).

The small-scale RTG breadboard was tested up to a corrected measured thermal input power of just above $80 \mathrm{~W}$, simulating the power output of an $\mathrm{Am}_{2} \mathrm{O}_{3}$ fuel pellet of the same volume as the heat source module. Electrical heating was used in this design and in subsequent generations of systems tested in the lab and reported in this paper. The use of electrical heating in developing radioisotope power is a standard approach; some examples in the literature of small scale electrically heated systems based on plutonium fuel include the systems developed by Woods et al. (2006) and Balint and Emis (2006). The system initially delivered a maximum electrical power output of $3.46 \pm 0.21 \mathrm{~W}$, corresponding to an overall system efficiency of $4.20 \pm 0.53 \%$. This maximum performance was achieved with two $\mathrm{Bi}_{2} \mathrm{Te}_{3}$ thermoelectric generator modules (consisting of 161 couples each) installed in conductive coupling to the aeroshell in a vacuum environment. Thermal efficiencies for the RTG of $73 \% \pm 13 \%$ were calculated based on experimental results. Switching to TEGs manufactured using bismuth telluride (Williams et al. 2014) materials produced via spark plasma sintering produced comparable results to standard materials whilst the performance for the $0.2 \mathrm{wt} \% \mathrm{~B}_{4} \mathrm{C}$ doped TEGs (Williams et al. 2014) is notably higher and meet the $5 \%$ total system efficiency target, with $4 \mathrm{~W}$ generated for $80 \mathrm{~W}$ of thermal power input. This was primarily driven by the lower electrical resistance values of these modules and the addition of the gold-plated heat shield improving the overall thermal efficiency of the system. The electrical resistance at peak power for two $0.2 \mathrm{wt} \% \mathrm{~B}_{4} \mathrm{C}$ doped TEGs operating in series was $35 \Omega$ as opposed to $60 \Omega$ for the very first generation of TEGs produced. In general, delta-T values of between $160{ }^{\circ} \mathrm{C}$ and $170{ }^{\circ} \mathrm{C}$ were obtained. The most significant conclusion was that at least equivalent performance could be obtained from a polycrystalline thermoelectric material produced by spark plasma sintering. These materials have much improved mechanical performance compared to conventional thermoelectric materials, giving the potential for increased reliability and reduced risk.

\subsection{Evolution in RTG Architecture: 2nd Generation Design}

The switch to bismuth telluride and the lower operating temperatures of americium presented the challenges of scaling power output, maintaining a modular design and keeping 


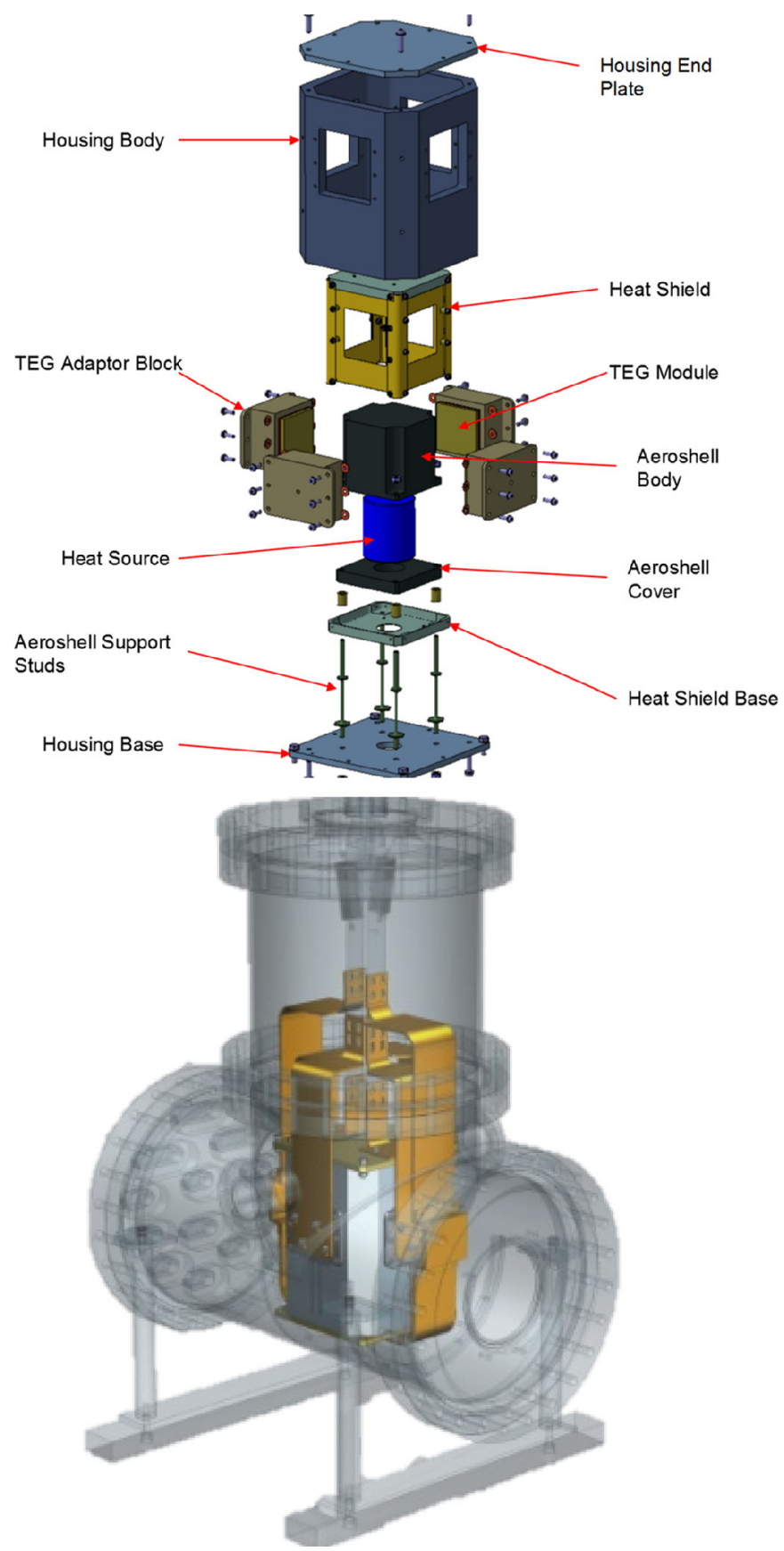

Fig. 12 (Top) The first lab-based system focused on an on-axis fuel design to minimise the heat source volume. The electrically heated unit was designed to deliver $80 \mathrm{~W}$ of thermal output and $4 \mathrm{~W}$ of electrical output. (Bottom) This unit was not hermetically sealed and was designed to be tested in a vacuum chamber. The system was originally designed to be cooled via a thermally controlled cold finger. The vacuum chamber was designed to be filled with argon at atmospheric pressure to simulate operation in deep space and planetary surfaces. (Bottom) A rendition of the first prototype system with external radiator in a flight like configuration 


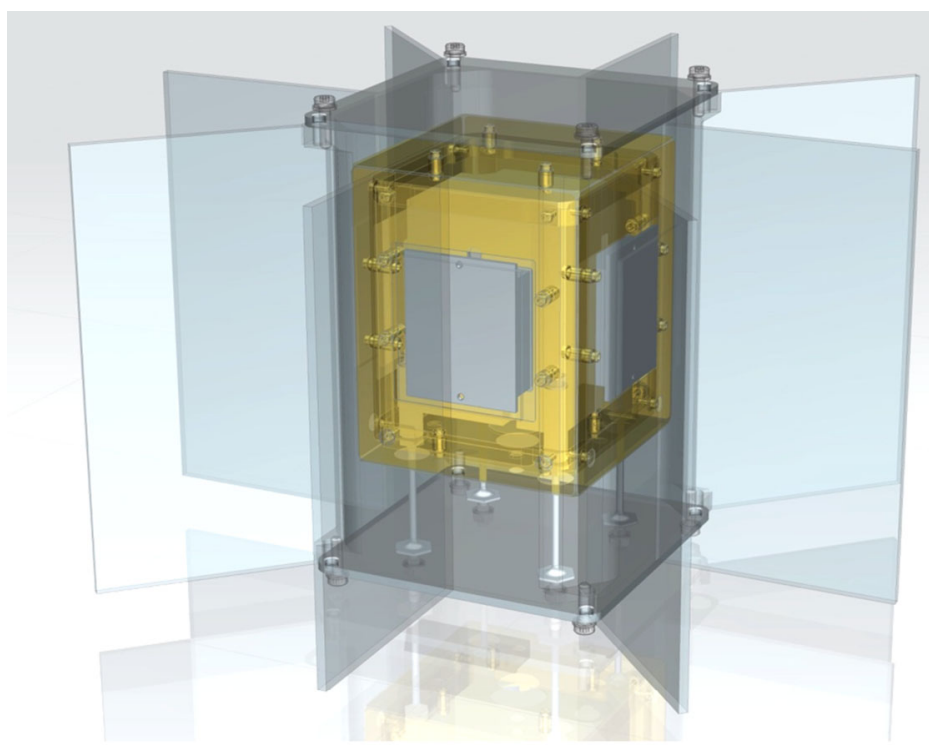

Fig. 12 (continued)
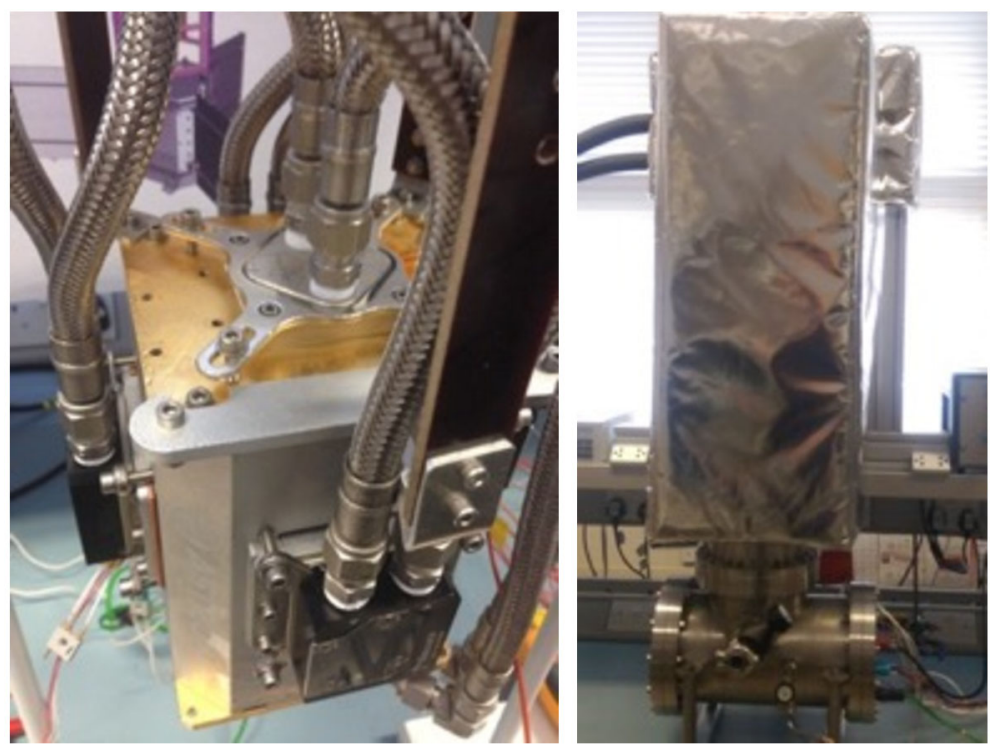

Fig. 13 The cooling system upgrade from cold finger to pumped fluid loop with cooling plates. The pipes feeding the fluid to the cooling plates are shown on the left. The system under a shroud is shown on the right

the overall heat source volume as small as possible without significantly increasing the overall dimensions of the RTG unit. Early prototype testing (see Sect. 5.2) outlined that a 5\% total system efficiency was a credible target. 


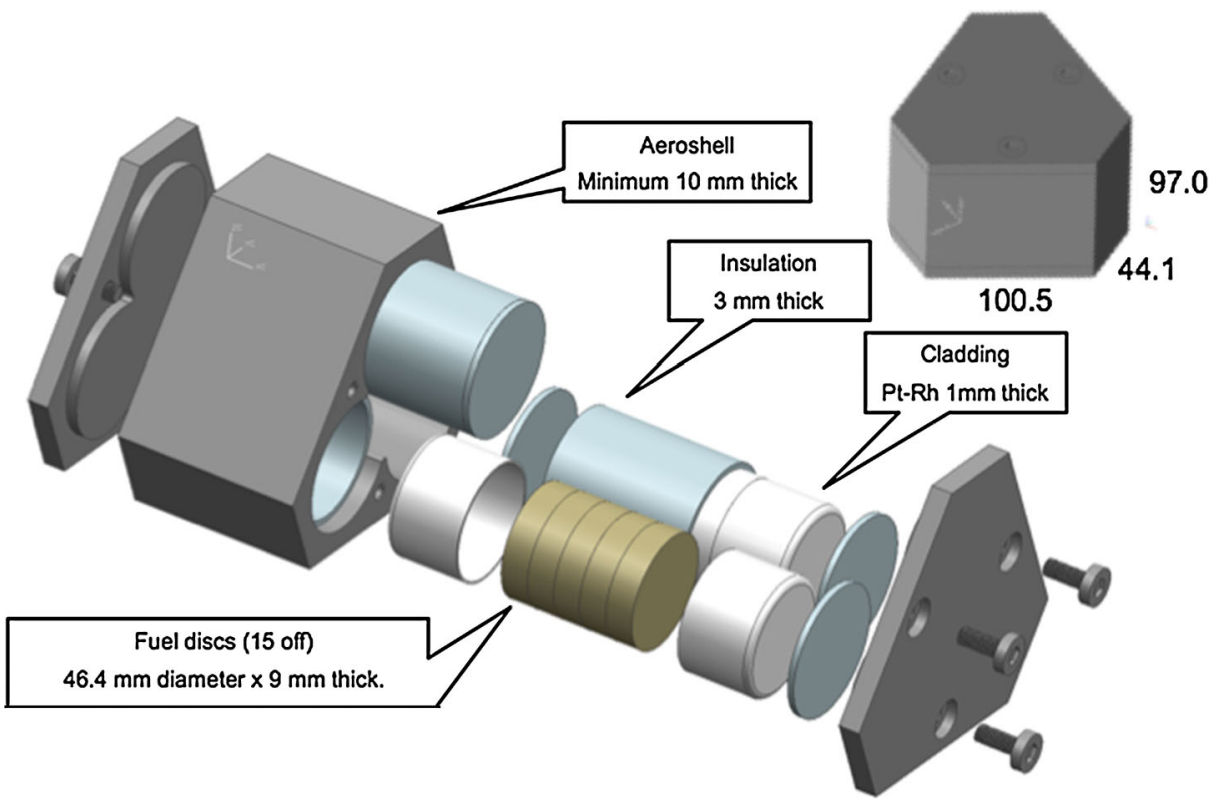

Fig. 14 The $200 \mathrm{~W}$ heat source consisted of a cloverleaf distributed fuel clad arrangement with $\mathrm{Am}_{2} \mathrm{O}_{3}$ fuel discs, $1 \mathrm{~mm}$ thick platinum-30\%rhodium cladding, insulation layer and outer carbon-carbon composite aeroshell. The dimensions are also shown

Although the overall specific power decreased as a consequence of a maturing design and the utilisation of bismuth telluride, the benefits of being able to use commercially available standard materials for these systems was significant. This approach increased feasibility and had positive implications in operational lifetime. Any specific power losses as a consequence were accepted as mitigating development risk.

Central to the evolution in the architecture was the heat source design. Migrating away from an on-axis heat source allowed the design team to scale the heat output by focusing on a six-sided geometry as shown in Fig. 13. This design would ensure that the volume occupied by the fuel was minimised. The six-sided heat source structure with a distributed cloverleaf fuel clad architecture increased the thermal power output to $200 \mathrm{~W}$ and with an assumed 5\% total system efficiency (based on earlier testing), a $10 \mathrm{~W}$ electric target was set for this design. The dimensions of the first-generation design of the six-sided heat source and the characteristics of the fuel cladding are shown in Fig. 14. This was later replaced with the second generation and current design outlined in Fig. 6.

The RTG that resulted from this design (see Fig. 15) retained some of the features of the early design. The TEG geometry was unchanged, the multilayer gold-plated heat shield was retained, the spring-loaded mounting concept for the TEGs and support struts for the heat source were also retained. However, additional significant changes were made. The radiator structure was designed to be a vacuum chamber with O-ring seals on radiator fins, lid and base. Feedthroughs for interfacing to the TEGs and for thermal sensors were vacuum compatible. Larger sides of the heat source were insulated to minimise losses and shorter sides of the heat source were used for making contact with the TEGs. The system was also designed to be operated under argon cover gas. Mechanical design and thermal models were used to understand the performance of the system and these are reported by Barco et al. (2018a, 2019b). 

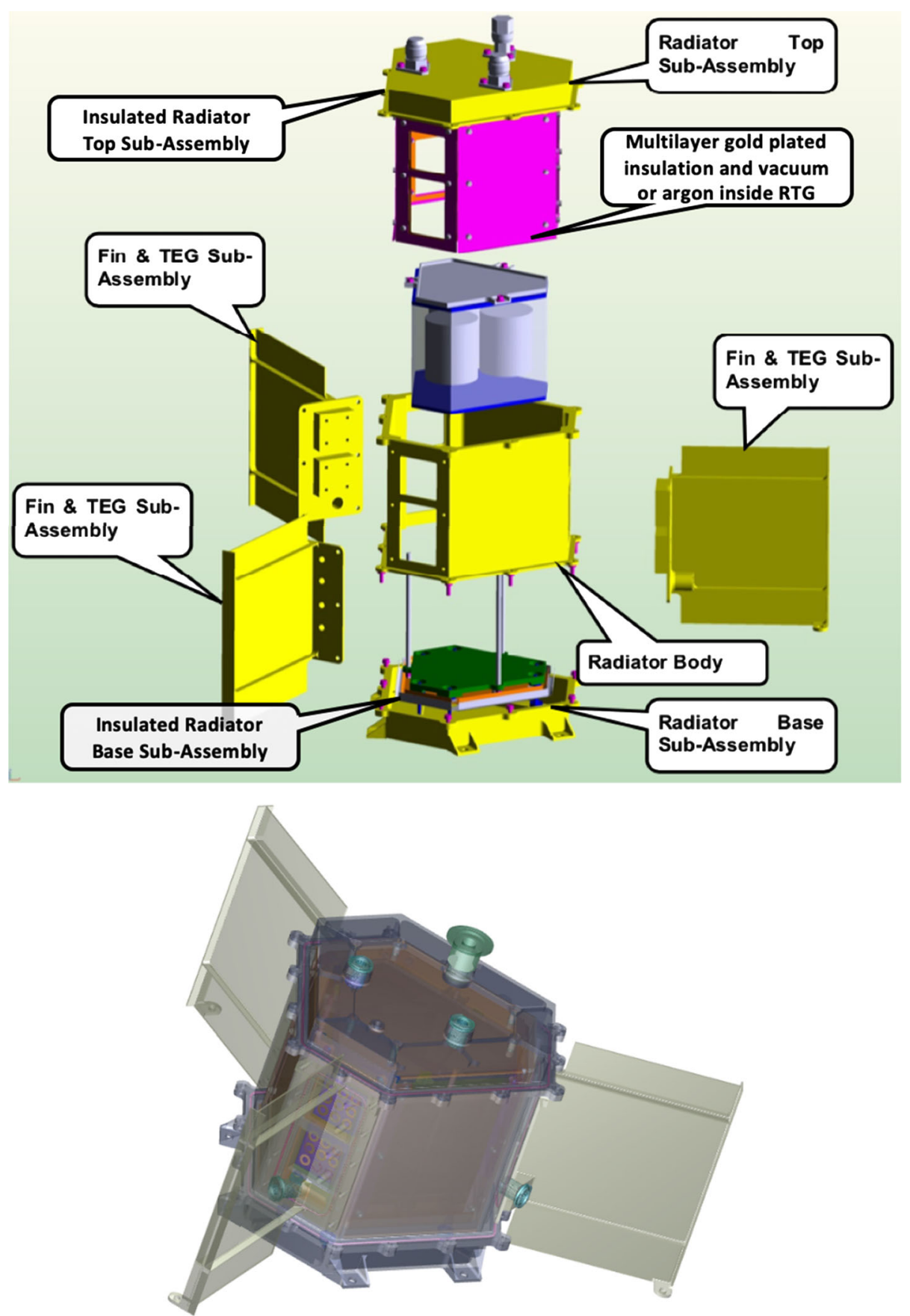

Fig. 15 (Top) Expanded view of the RTG design with labelled subassemblies. (Bottom) An image of the fully assembled system showing pumping port and electrical feedthroughs 
The design of the heat source is such that top, bottom and large sides of the six-sided structure are insulated by using a multilayer gold-plated rigid structure with very low emissivity that is specifically designed for this system. The combination of this design of multilayer insulation and vacuum inside the chamber ensures that the heat flows mainly through the thermoelectric generators in contact with the heat source. Alternatively, Ar can be inserted in the chamber which still provides a degree of insulation but is not as effective as vacuum. The lower operating temperature of the system also reduces the radiation losses compared with higher power systems that operate at higher temperatures.

\subsection{Building the Elegant Breadboard of the 10 W Electric RTG}

The scaling of a functional lab system from initial concept to a more representative system is shown in Fig. 16. This system was fully tested. Tests included bench testing and testing in a representative space environment simulator at Leicester. In addition, laser Doppler vibrometry testing (Barco et al. 2018a, 2018b, 2019b), using specialised facilities at the University of Leicester, enabled the team to verify the mechanical models produced for the system without resorting to destructive conventional vibration tests. This RTG system incorporated six thermoelectric modules supported by the fins of the radiator. Tests with argon cover gas were also executed. Notable features of this system include (see Fig. 16):

- Carbon-carbon composite aeroshell for the heat source.

- Multilayer gold-plated heat shield.

- System is a sealed vacuum chamber with pumping port and electrical feedthroughs for heating, thermal sensing and TEG outputs.

- System can be operated under cover gas.

- System mass of $9.4 \mathrm{~kg}$ excluding the liquid-cooling plates.

- Pumped fluid loop cooling system with cooling plates attached to the radiator.

\subsection{Refinement of the RTG Architecture: 3rd Generation Design}

The architecture was further refined to reflect the updated heat source design that is shown in Sect. 3.1. The design (as shown in Fig. 17) was updated to reflect the following:

- The work by Watkinson et al. (2017b) suggests that a smaller diameter fuel form compared to early designs is preferred in order to ensure the successful consolidation of americium ceramic fuel. This is reflected in the clad design shown in Sects. 2.2 and 3.2.

- Standardisation of fuel clad manufacturing processes across RHU and RTG systems.

- The work by Vigier et al. (2018) also suggests that the fuel form is likely to be a mixed oxide of americium and uranium-based fuels where the latter could comprise $15 \%$ of the total fuel mass.

- The need to potentially accommodate more fuel mass while constraining the heat source volume.

- A larger radiator to ensure that TEG cold side temperatures are in line with the ZT curve of bismuth telluride.

Thermal analyses of this design (Table 8, Fig. 18) show temperatures in line with the requirements of the baseline thermoelectric generator design described in Sect. 4.

The heat source itself has been modelled in detail, some of which is shown in Fig. 18. The top panel of Fig. 18 provides an indication of the heat source temperatures at the interfaces with the TEGs. The TEG interface temperatures in this model are at around $190{ }^{\circ} \mathrm{C}$ and 

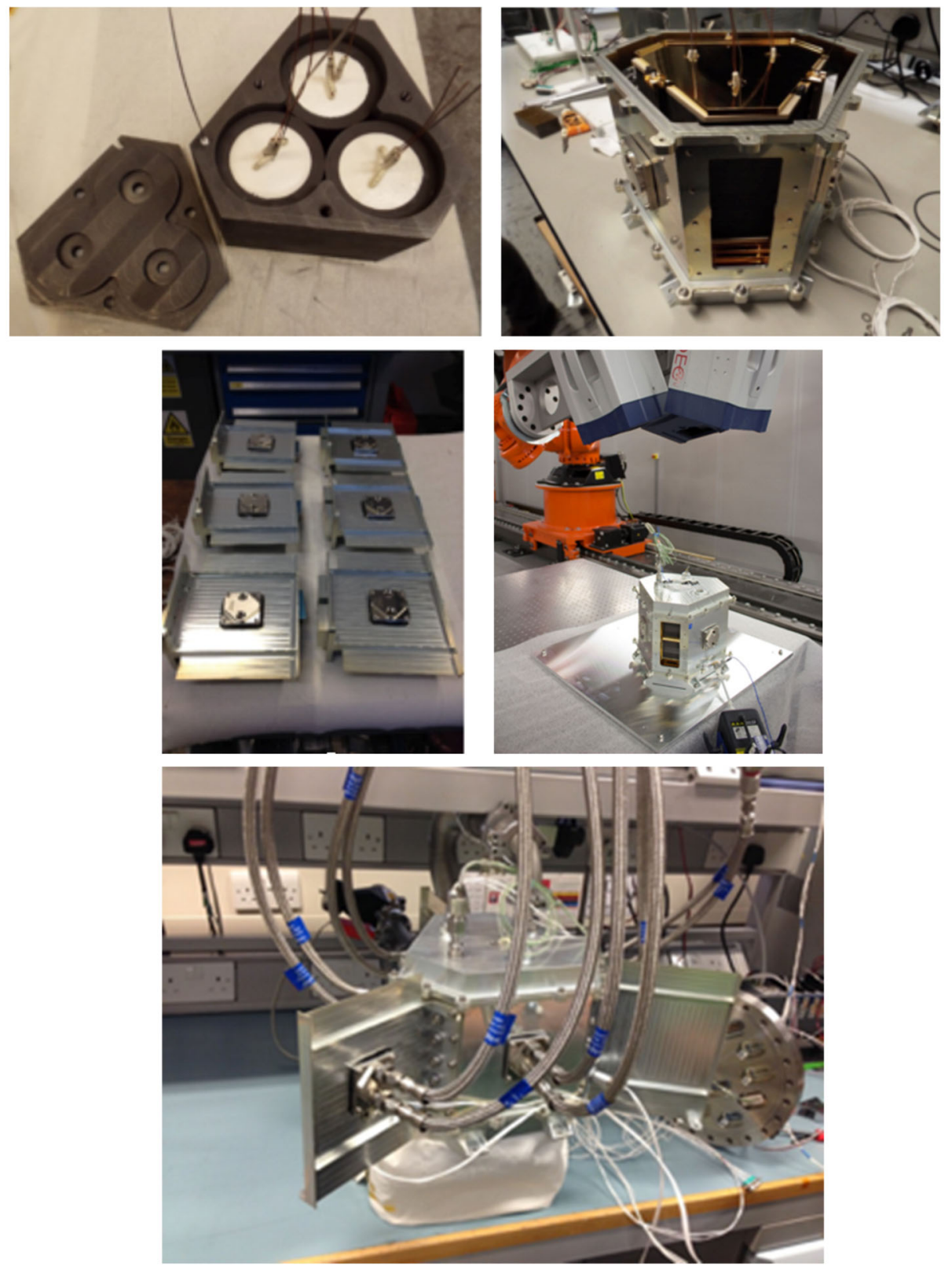

Fig. 16 (Top left) The $200 \mathrm{~W}$ heat source consisting of carbon-carbon composite aeroshell with electrical heaters. (Top right) The radiator body surrounding the heat source and heat shield subassemblies. (Middle left) Radiator fins. (Middle right) System undergoing vibration testing using laser Doppler vibrometry. (Bottom) Fully assembled system ready for testing on the bench

can vary up to $225^{\circ} \mathrm{C}$ depending on how the coupling efficiencies within the structure are modelled. The detailed images of the internal parts of the heat source are not provided due to the proprietary nature of these designs. 

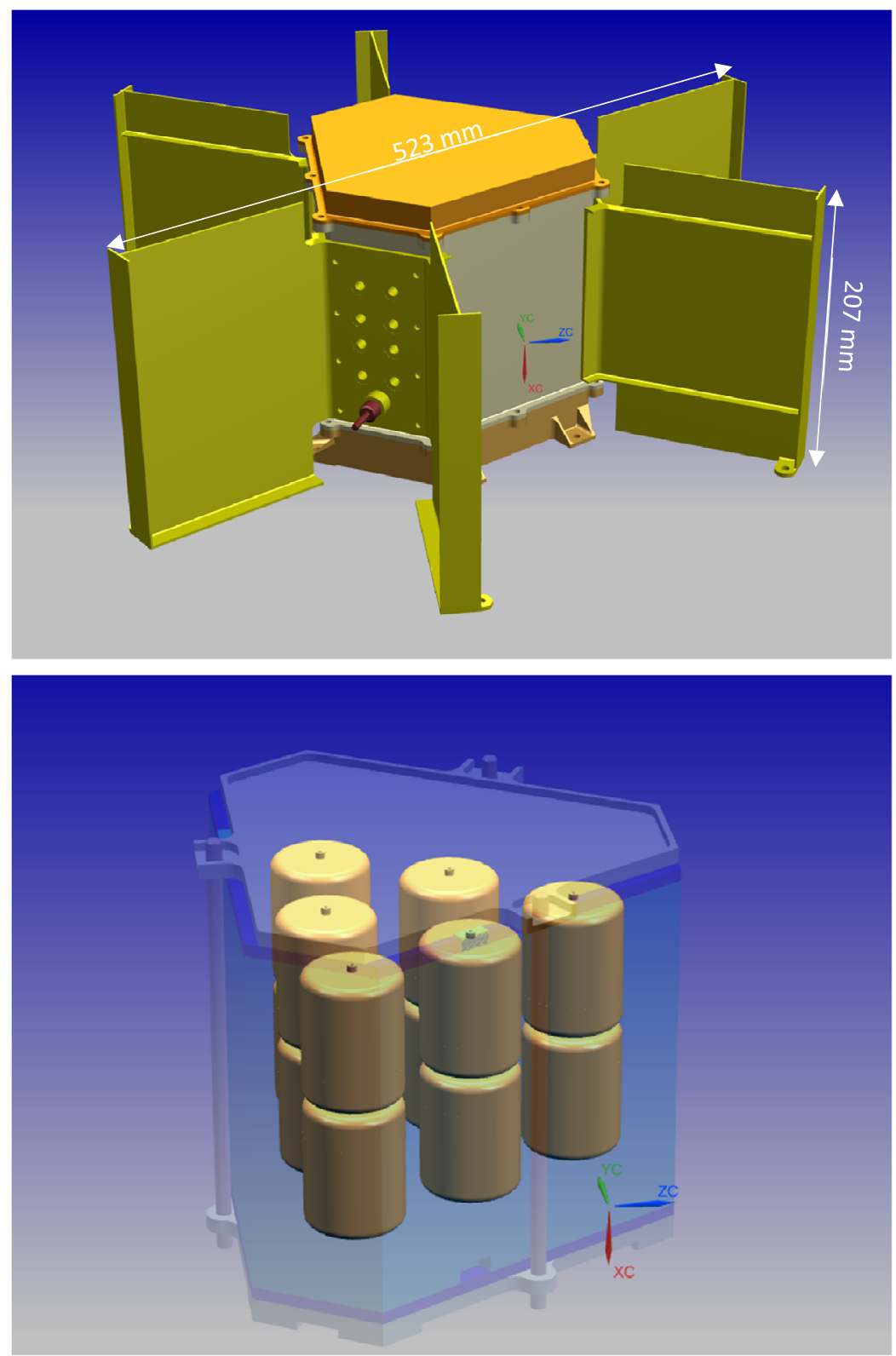

Fig. 17 The new RTG design has a larger radiator (6 fins rather than 3 ) and 12 fuel clads to deliver approximately $200 \mathrm{~W}$ of thermal power from a uranium-stabilised americium oxide

When analysing the results, some interesting points were noted. The radiator body is hotter than the fins: since at least $75 \%$ of the total heat supplied flows through the TEGs and then to the fins, the fin average would be expected to be at a higher temperature. This could be caused by the choice of thermal coupling coefficients in the model, or by the design itself. The fuel is now closer to the larger sides of the aeroshell, which means that more heat is 

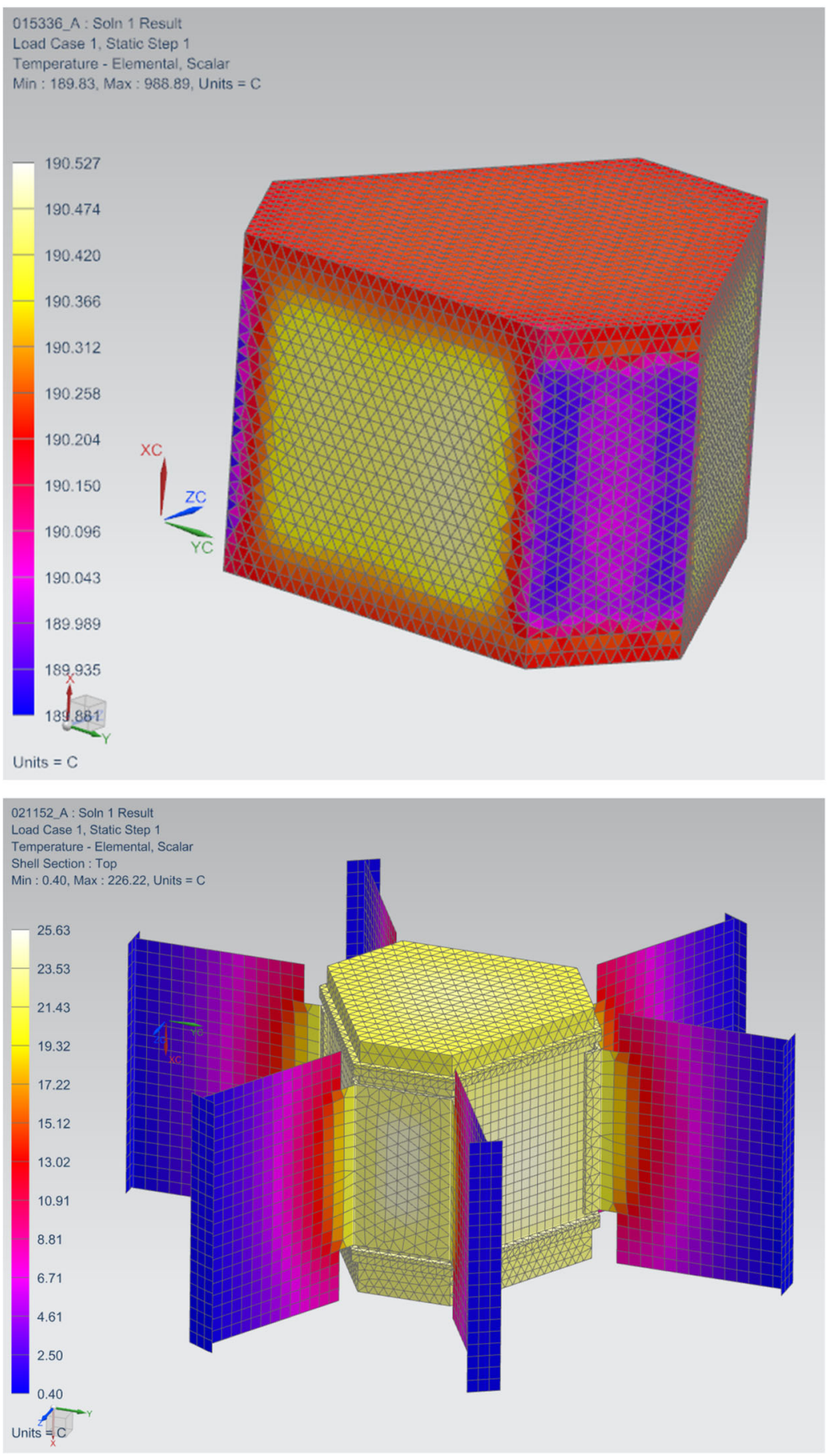

Fig. 18 (Top) Thermal analysis of the heat source showing the temperature distribution across the external parts of the heat source structure. (Bottom) The temperature distribution from the finite element model of the system 
Table 8 Results from the finite element thermal analysis for the new design

${ }^{\mathrm{a}}$ The hot side of the thermoelectric generators can vary depending on the coupling efficiencies in the model. In the latest design this is $224^{\circ} \mathrm{C}$ but can range between $190^{\circ} \mathrm{C}$ and $225^{\circ} \mathrm{C}$

${ }^{\mathrm{b}}$ Similarly the TEG cold side can also vary between $10^{\circ} \mathrm{C}$ and $25^{\circ} \mathrm{C}$ in the thermal model

\begin{tabular}{ll}
\hline Components & $\begin{array}{l}\text { Temperature } \\
{\left[{ }^{\circ} \mathrm{C}\right]}\end{array}$ \\
\hline Aeroshell & 226 \\
TEG hot face & $224^{\mathrm{a}}$ \\
TEG cold face & $25^{\mathrm{b}}$ \\
Radiator body (average) & 23 \\
Fin (average) & 16 \\
Fin (tips) & 0 \\
Radiator \& fin (average) & 21 \\
\hline
\end{tabular}

likely to be transferred to the radiator body directly and not via the TEG modules. This could also suggest that the fin conductivity is too low in the model and that losses are higher than expected. This analysis outlines a likely worst-case scenario that will need to be confirmed by measurement. Particularly given the higher thermal efficiencies obtained experimentally with earlier designs suggest that $80 \%$ to $90 \%$ of the heat flows through the TEGs. Measurements based on the latest design are planned in future phases of the programme once the build of this new model has been completed.

\section{Designing and Building the RHU}

Three generations of RHU flight design have been produced, each building and improving on previous designs. Two prototype devices reflecting the flight designs have been built and tested. In addition, mechanical models have been developed for vibration testing and some safety studies. The current TRL of the RHU system is 4 (i.e. breadboard functional verification in a laboratory environment). The University of Leicester team is currently working on a further iteration of the flight design and is in the process of building a flight system to TRL 5 (i.e. breadboard critical functional verification in a relevant environment) on the new ESA ISO scale (ECSS 2017). The plan is for the new RHU system to be completed in 2020. A roadmap to develop a flight-ready RHU by 2025 or 2026 has been developed in collaboration with ESA, which would be compatible with an outer solar system mission target of around 2030.

The RHU design architecture is highlighted in Sect. 2 and details of the mechanical and thermal performance are provided in detail by Barco et al. (2018b). Models developed by Barco et al. (2018b, 2019a) for both the thermal and the structural analysis of the RHU assume good contact between the different layers of the heat source under operating conditions. Rapid heating during assembly would result in a compression of all the layers due to thermal expansion. It is not pre-defined how an ESA RHU would be thermally integrated in a spacecraft; therefore, three cases were analysed in order to cover all the possible configurations of the RHU installation on a spacecraft:

- Radiation to the internal spacecraft environment and conduction via a thermal management interface.

- Conduction only via a thermal management interface with the spacecraft, which has a temperature of $20^{\circ} \mathrm{C}$. This would require insulation around the RHU to minimise radiation.

- Radiation only to the internal spacecraft environment $\left(20^{\circ} \mathrm{C}\right)$. 

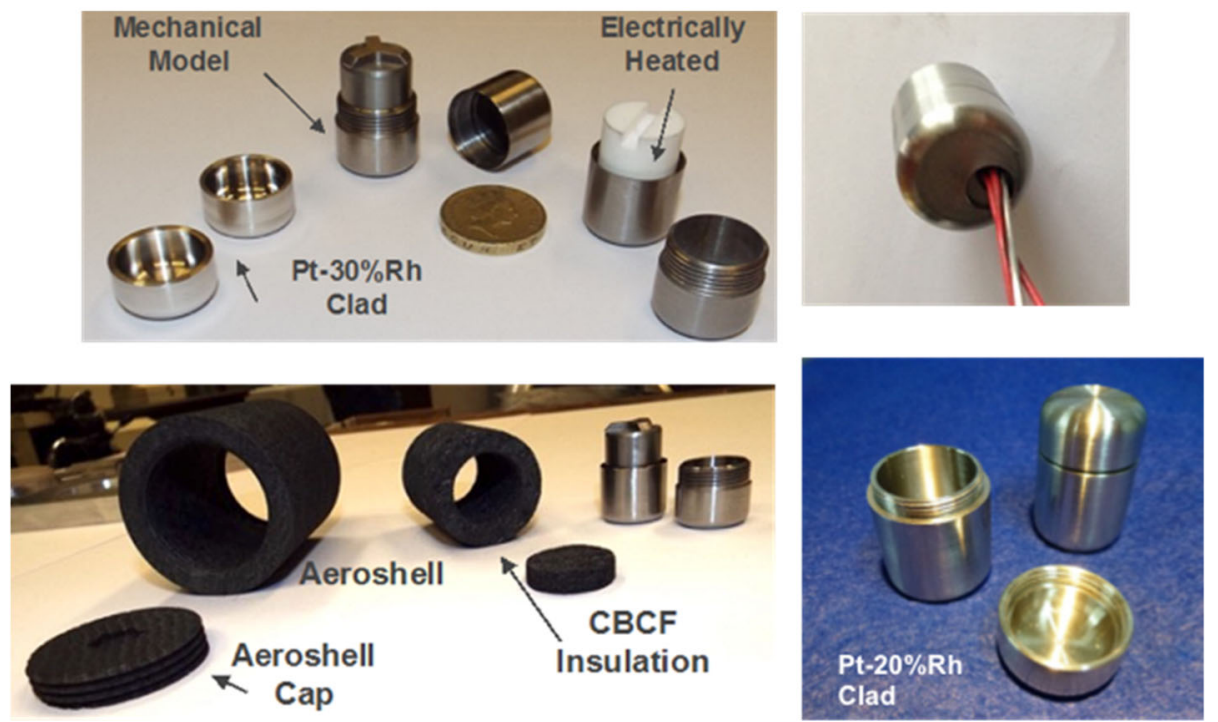

Fig. 19 The components that make up the RHU system including aeroshell, insulation, heat source (electrically-heated), cladding in Pt-30\%Rh and threaded Pt-20\%Rh

Table 9 RHU thermal modelling results

\begin{tabular}{lll}
\hline Case & $\begin{array}{l}\text { Aeroshell temperature } \\
{\left[{ }^{\circ} \mathrm{C}\right]}\end{array}$ & $\begin{array}{l}\text { Fuel temperature } \\
{\left[{ }^{\circ} \mathrm{C}\right]}\end{array}$ \\
\hline Conduction \& Radiation & 24 & 190 \\
Conduction & 25 & 191 \\
Radiation & 67 & 233 \\
\hline
\end{tabular}

Table 9 reports the maximum calculated temperatures for the centre of the fuel and the aeroshell, assuming a steady-state analysis.

Higher values were obtained for the radiation case, while the other two cases had almost the same temperature values: in the combined heat transport case, the proportion of heat transferred by radiation was small.

Figure 19 shows the different components of the RHU that were sourced to produce a mechanical model and an electrically heated model. Figure 20 shows the test fixture used to test the RHU and generate the results shown in Table 10. These models include the following components:

- A carbon-carbon composite aeroshell.

- Carbon-bonded-carbon fibre $(\mathrm{CBCF})$ insulation layer that was $6 \mathrm{~mm}$ thick rather than prescribed $3 \mathrm{~mm}$ thickness, due to availability and manufacturability.

- Electrical heaters for the thermal tests (using conventional resistive heating in a ceramic support structure), housed initially in a substitute stainless steel cladding structure and subsequently in Pt-20\%Rh.

- Molybdenum (with a similar density to americium oxide at $85 \%$ of theoretical density) as a mass dummy for the fuel in a mechanical RHU prototype, utilising Pt-30\%Rh cladding.

Initially a Pt-30\% Rh clad was sourced and this was replaced with an updated Pt-20\% Rh threaded clad. The migration from Pt-30\%Rh to Pt-20\% Rh was primarily due to cost and 
Fig. 20 The RHU (black cylindrical structure) mounted against an Al plate simulating a spacecraft interface in conductive operation mode. The thermal management system consisting of pumped fluid loop and heat exchanger was used to control the temperature of the interface. The assembly was operated in vacuum and in a nitrogen atmosphere

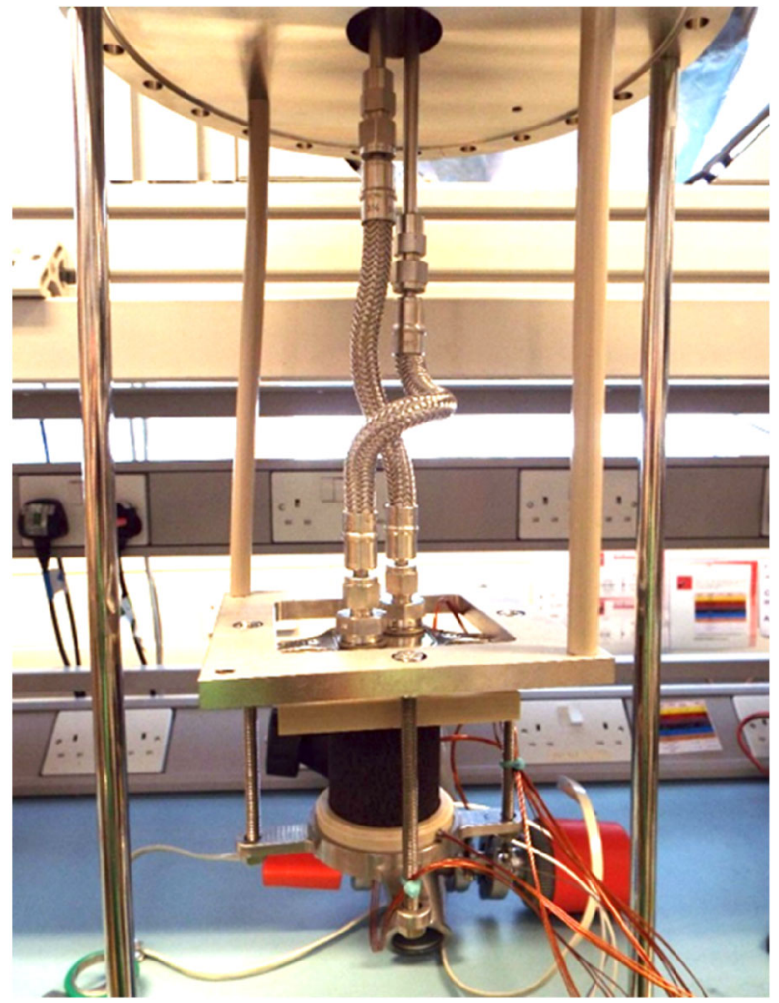

availability, but there is no indication in the open literature that a $20 \% \mathrm{Rh}$ alloy is not equally suitable. Notably, Pt-20\%Rh was used as one of the cladding layers (Angelo and Buden 1985) in the SNAP-19 US RTG design (Hula 2015). Further detailed evaluation of the materials under non-operational conditions is currently underway and will be reported at a future date.

\section{Testing Radioisotope Power Systems}

\subsection{Testing the RHU}

The electrically-heated prototype was tested in various configurations at constant power. A spacecraft interface simulator was developed specifically for testing: the heat produced was managed via an Al-based interface plate, a cooling block attached to the plate and a pumped fluid loop. The system was tested in various configurations as shown in Table 10. Thermal sensors were inserted in various points in the system: in the heat source (crucible) near the electrical cartridge heater; embedded in the aeroshell sleeve; embedded in the aeroshell cap; in the base plate; near the insulation; and on the bracket. The thermal sensors measured the temperatures reached by the components, under different test conditions. The results shown are for the tests carried out with the Pt-20\% Rh clad.

\subsection{Testing the RTG}

The experimental testing of both RTG systems is summarised in Fig. 21. The results show how the evolution from the small-scale $4 \mathrm{~W}$ unit and improvements in system-level design 
Table 10 Results for the RHU thermal tests

\begin{tabular}{llllr}
\hline & $\begin{array}{l}\text { Power } \\
{[\mathrm{W}]}\end{array}$ & $\begin{array}{l}\text { Aeroshell temperature } \\
{\left[{ }^{\circ} \mathrm{C}\right]}\end{array}$ & $\begin{array}{l}\text { Fuel temperature } \\
{\left[{ }^{\circ} \mathrm{C}\right]}\end{array}$ & $\begin{array}{l}\text { Plate temperature } \\
{\left[{ }^{\circ} \mathrm{C}\right]}\end{array}$ \\
\hline General case & 3 & 32 & 368 & -12.5 \\
& & 46 & 403 & 5 \\
Conduction in vacuum & 3 & 55 & 365 & -10 \\
& & 63 & 374 & 5 \\
Conduction in a nitrogen & 3 & 20 & 88 & -10 \\
atmosphere & 31 & 99 & 5 \\
Radiation in vacuum & 3 & 68 & 440 & 15 \\
& 3.2 & 74 & 455 & 20 \\
\hline
\end{tabular}

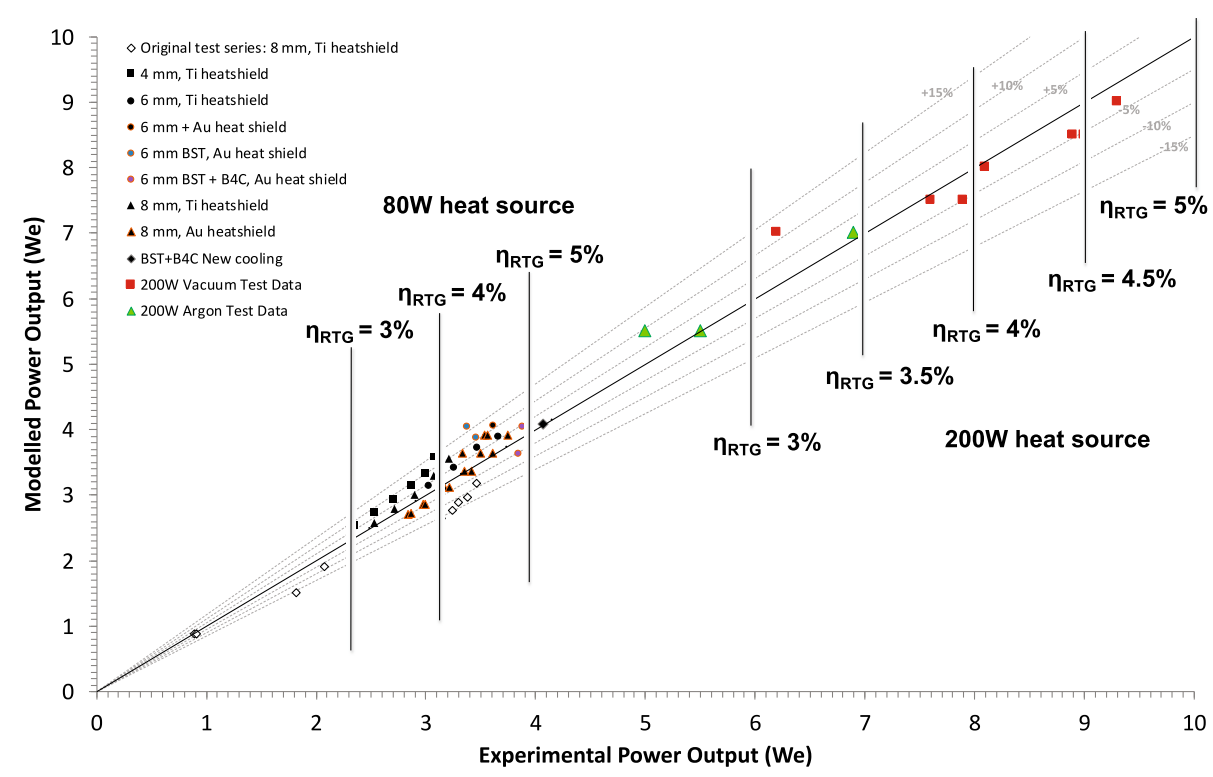

Fig. 21 Experimental and modelling results from testing both the $80 \mathrm{~W}$ thermal and $200 \mathrm{~W}$ thermal RTG systems

and TEG production resulted in achieving a total system-level conversion efficiency of 5\%. Scaling to the $200 \mathrm{~W}$ thermal heat source showed that, with a hot side of $199^{\circ} \mathrm{C}$ and a cold side of $21^{\circ} \mathrm{C}$, a total power output of between $9.1 \mathrm{~W}$ and $9.3 \mathrm{~W}$ electric was feasible, providing a total system level efficiency of $4.55 \%$ to $4.65 \%$. These data corroborated calculations carried out prior to testing. Additional improvements could be made to both the system and the TEGs in order to improve the overall efficiency, and these are a focus of current ongoing work. Adding argon at 1 bar to the system did not significantly degrade the performance of the RTG: the data show that power output dropped to around $7 \mathrm{~W}$ electric and overall system efficiency decreased to $3.5 \%$. Using a cover gas at 1 bar pressure is seen as worst case; a lower argon pressure would have a yet lower impact on power output. The total mass of $9.4 \mathrm{~kg}$ and the power output imply that the $1 \mathrm{~W} / \mathrm{kg}$ specific power value remains applicable when scaling up from the $4 \mathrm{~W}$ electric unit to the $10 \mathrm{~W}$ electric unit. 


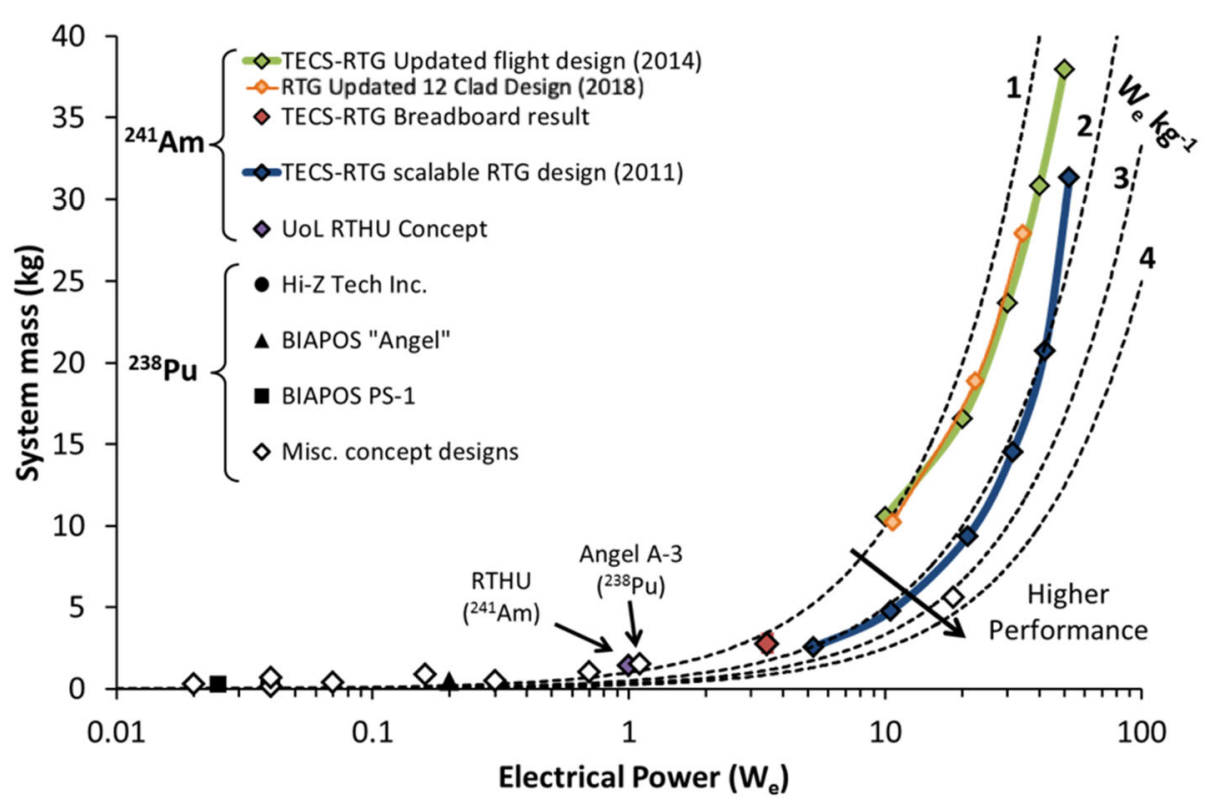

Fig. 22 Summary of the small-scale RTG performance including the more recent $10 \mathrm{~W}$ electric unit

Electrically, each pair of TEGs on each radiator fin operated in series, with the three series pairs connected in parallel. This configuration would provide redundancy at the system level, with failure of a TEG not resulting in total failure at system level. During testing, TEGs with similar electrical and thermoelectric characteristics were connected in series on each fin, and three separate external loads were utilised, each connected to one series pair. This allowed maximum flexibility in the testing of TEGs and the investigation of optimum configurations. Voltage output levels were around $11 \mathrm{~V}$ in vacuum and $9.5 \mathrm{~V}$ under Ar gas. Currents were approximately $850 \mathrm{~mA}$ and $740 \mathrm{~mA}$ respectively, for the three series pairs combined.

\subsection{Evolution in Specific Power with Design and Test Data}

In the following section, a comparison is made between the specific power estimates in the early phases of the project and those emerging from the latter phases. The specific power values as a function of power output of the updated designs of the $200 \mathrm{~W}$ heat source $10 \mathrm{~W}$ electric RTG are included in the figures below. The basis for the graph in Fig. 22 is given in detail by Williams et al. (2011).

Solid symbols (with no connecting lines) represent studies that historically have been subject to hardware demonstration or higher technology readiness levels and are described by Williams et al. (2011). Concept designs (unfilled symbols) are those that appear to be paper-based design studies and refer to concepts based on small-scale RTG systems and are included for comparison. These concepts are described in detail by Pustovalov (2007), Abelson et al. (2004) and Williams et al. (2011). The dotted performance contours on the graph show mass-specific electrical power. The blue line with solid blue symbols and the green line with solid green symbols respectively refer to the outputs from the early system architecture studies with the fuel on-axis and the design studies with the $200 \mathrm{~W}$ thermal heat source completed in 2014. The red symbol refers to the breadboard or first RTG lab system 
delivering $4 \mathrm{~W}$ of electrical power. The orange line with solid orange symbols refers to the most recent updated RTG system based on the $200 \mathrm{~W}$ design with 12 clads rather than 3 clads, which was completed in 2018. The plot shows that exceeding the $2 \mathrm{~W} / \mathrm{kg}$ specific power level is challenging with an Am-based power system.

\section{Conclusion}

The ESA radioisotope power systems programme has resulted in the evolution of RTG system design from a $4 \mathrm{~W}$ electric system to a $10 \mathrm{~W}$ electric system. Standard heat source designs for RTG and RHU application have been produced to form the building blocks of European RTGs and RHUs that would be deployed in space. In the case of the RTG systems developed, mass-specific power levels of $1 \mathrm{~W} / \mathrm{kg}$ have been demonstrated. Mechanical and thermal models produced correlate effectively with experimental tests. Thermoelectric production methods have been established in the UK with the capability to produce bespoke thermoelectric modules based on bismuth telluride technology. Testing on the bench and in a space environment simulator was carried out. Two $10 \mathrm{~W}$ electric lab prototypes were successfully tested, incorporating carbon-carbon composite and graphite aeroshells respectively. Additional testing of subcomponents (cladding, aeroshell materials) under nonoperational conditions was successfully completed and reported by Ambrosi et al. (2019). Extended operation testing of TEGs was successfully completed (Mesalam et al. 2019). Irradiation of TEGs was also successfully completed (Mesalam et al. 2019). Extended lifetime and radiation testing suggest that bismuth telluride TEG technology is robust partly due to lower operating temperatures of these RTG systems.

A scaled $50 \mathrm{~W}$ electric unit is currently being designed, based on optimisation of the $10 \mathrm{~W}$ electric unit as the system level building block. The focus is less on specific power but rather optimisation of the power output, reliability and longevity of the building block (i.e. the $10 \mathrm{~W}$ electric unit). This approach will reduce the development time and control the cost for larger RTGs applicable to missions requiring several hundred Watts of electrical power. This approach will also make building and assembly of RTG systems with radioisotope fuel more straightforward.

The RHU for the ESA RPS programme has been designed and tested in a representative configuration and environment. The RHU is a $3 \mathrm{~W}$ thermal unit with an overall mass of $200 \mathrm{~g}$, giving a specific thermal power of $15 \mathrm{~W} / \mathrm{g}$. This is a competitive design and can be operated in a number of modes: conduction only, radiation only, or both conduction and radiation.

Materials for the RTG and RHU laboratory test units reflect the flight designs and include carbon-carbon composites for the aeroshell and platinum alloy fuel cladding. A frit vent has been also added in the design of the cladding for both RTG and RHU systems, to allow the helium and the oxygen released by the fuel to flow out of the inner containment and, therefore, avoid stresses on the clad. This frit development is currently underway and will feature in future prototype models.

Concerning the fuel development, pellet sintering was performed both on surrogate materials and on americium oxide. The stabilisation of cubic americium oxide with uranium was proposed to improve the ceramic properties.

The next steps in the programme (already underway) include:

- To improve the characterisation of the Pt- $20 \% \mathrm{Rh}$ thermal and mechanical properties, especially at high temperatures.

- Although successful clad welding trials were recently completed, data and welds are still being analysed and further work on clad welding is required. 
- To perform new launch failure and Earth re-entry performance studies.

- To perform new analyses for the frit vent design (choice of porosity and frit particle sizes), also keeping in mind the need for manufacturing and testing.

- To perform further vibration tests (to find natural frequencies, and through a wider vibration spectrum).

- To further develop the americium fuel ceramic production, achieving better control of the microstructure and the open porosity, investigating ${ }^{16} \mathrm{O}$ enrichment to decrease neutron production, determining the minimum amount of uranium needed to stabilise the cubic oxide form in freshly purified ${ }^{241} \mathrm{Am}$ and scaling up the ceramic production.

- To perform interaction tests between americium fuel ceramic and the cladding material.

The RTG systems outlined in this paper could be easily adapted to provide power in challenging environments on Earth such as deep geological repositories for storing nuclear waste, deep sea applications or in locations where a deployable long-lived power system is required. The systems outlined in this paper and variations of these could be modified and adapted to deploy in terrestrial environments. RHUs could be used to make very small RTG systems delivering power outputs between $100 \mathrm{~mW}$ and $1 \mathrm{~W}$ electric.

The ESA radioisotope power systems programme has been active since 2009, when the first radioisotope selection studies were initiated. Since then, a series of projects have advanced the development of heat sources and heat-to-electricity conversion technologies that could enable a range of ESA missions in the latter half of the 2020s and into the 2030s. The University of Leicester team has been working on three aspects of the programme since 2010: radioisotope thermoelectric generators (RTGs); radioisotope heater units (RHUs); and supporting the National Nuclear Laboratory in developing the americium fuel form. The technology programme to date has advanced these technologies to TRL 4 or "component and/or breadboard functional verification in laboratory environment". By the early 2020s, the maturity levels of these systems (i.e. RTGs and RHUs) will reach TRL6 on the ISO scale or "model demonstrating the critical functions of the element in a relevant environment", preparing the way for the utilisation of these systems in future missions. In the course of the TRL progression to date, the various system aspects and the radioisotope fuel work have converged into a programme where the different elements that make up space nuclear power systems can no longer be developed in parallel or isolation, given that each subcomponent has an impact on the overall system design and performance. This has resulted in the establishment of a unique community of partners across academia and industry as well as the identification of the facilities and infrastructure that have enabled ESA to establish a sustainable space nuclear power technology programme. Europe has all of the expertise and facilities to offer competitive radioisotope power solutions for both planetary science and deep space missions. In addition, the ESA programme has developed significant credibility on the international stage. This is embodied by the establishment of a network of international partners which has the potential to open up bilateral or multilateral exploration or scientific initiatives in the future.

Acknowledgements This work is funded by the European Space Agency. The authors would like to thank the UK Space Agency and in particular Sue Horne, Nick Cox, Elizabeth Seaman, Tony Mears, Helen Roberts, Libby Jackson. The results outlined in this programme would not have been possible without the support of Matthew Stuttard. The authors would also like to thank the team at the University of Dayton: Chadwick Barklay, Steven Goodrich, Christopher Whiting and Ronald Hoffman for their continued help and support. The authors like to thank Jan König and Martin Jägle from Fraunhofer IPM in Freiburg for their early work and collaboration on lead telluride thermoelectric generators. Robert C O'Brien whose PhD work sponsored by the Engineering and Physical Science Research Council contributed to the very early research at Leicester in this field. 
Publisher's Note Springer Nature remains neutral with regard to jurisdictional claims in published maps and institutional affiliations.

Open Access This article is licensed under a Creative Commons Attribution 4.0 International License, which permits use, sharing, adaptation, distribution and reproduction in any medium or format, as long as you give appropriate credit to the original author(s) and the source, provide a link to the Creative Commons licence, and indicate if changes were made.The images or other third party material in this article are included in the article's Creative Commons licence, unless indicated otherwise in a credit line to the material. If material is not included in the article's Creative Commons licence and your intended use is not permitted by statutory regulation or exceeds the permitted use, you will need to obtain permission directly from the copyright holder.To view a copy of this licence, visit http://creativecommons.org/licenses/by/4.0/.

\section{References}

R.D. Abelson et al., NASA Jet Propulsion Laboratory, JPL Pub 04-10 (2004). https://trs.jpl.nasa.gov/handle/ 2014/40856, accessed 30 August 2019

H.J. Allen, A.J. Eggers, in National Advisory Committee for Aeronautics Report, vol. 1381 (1957), p. 1125

R.M. Ambrosi, A. Barco, H. Williams et al., in Proceedings Nuclear and Emerging Technologies for Space Conference (American Nuclear Society, LaGrange Park, 2018), 978-0-89448-744-6

R.M. Ambrosi, E.J. Watkinson, A. Barco, R. Mesalam et al., in Proceedings IEEE Aerospace Conference (2019). https://doi.org/10.1109/AERO.2019.8742245

J.A. Angelo, D. Buden, Space Nuclear Power. Orbit, a Foundation Series (1985), p. 138

C.S. Arridge, N. Achilleos, J. Agarwal et al., Planet. Space Sci. 104, 122-140 (2014). https://doi.org/10.1016/ j.pss.2014.08.009

T.S. Balint, N.D. Emis, AIP Conf. Proc. 813, 327 (2006). https://doi.org/10.1063/1.2169209

A. Barco, R.M. Ambrosi, H.R. Williams et al. (American Nuclear Society), in Proceedings of Nuclear and Emerging Technologies for Space (NETS 2018), Paper 24177 (2018a). 978-0-89448-744-6

A. Barco, R.M. Ambrosi, H.R. Williams et al. (American Nuclear Society), in Proceedings of Nuclear and Emerging Technologies for Space (NETS 2018), Paper 24176 (2018b). 978-0-89448-744-6

A. Barco, C. Fongarland, R.M. Ambrosi et al., in Proceedings IEEE Aerospace Conference (2019a). https:// doi.org/10.1109/AERO.2019.8741744

A. Barco, R.M. Ambrosi, H.R. Williams et al., in Proceedings IEEE Aerospace Conference (2019b). https:// doi.org/10.1109/AERO.2019.8741786

G.L. Bennett, AIAA Pap. 2006(4191), 26-29 (2012). https://doi.org/10.2514/6.2006-4191

J. Birden, K. Jordan, US Patent 2,913,510 (1959). https://patentimages.storage.googleapis.com/1a/64/d3/ 0acea71016065f/US2913510.pdf, accessed 30 August 2019

A.M. Brandis, C.O. Johnston, in 45th AIAA Plasmadynamics and Lasers Conference Atlanta, GA (2014). https://doi.org/10.2514/6.2014-2374

T. Caillat et al., in Presented at Direct Thermal-to-Electrical Energy Conversion Conference, San Diego (2006). https://trs.jpl.nasa.gov/bitstream/handle/2014/40238/06-2720.pdf?sequence=1, accessed 30 August 2019

T.D. Chikalla, L. Eyring, J. Inorg. Nucl. Chem. 30, 133 (1968)

R.W. Detra, H. Hidalgo, J. Am. Rocket Soc. 31, 318-321 (1961). https://doi.org/10.2514/8.5471

M.S. El Genk, J-M. Tournier, Nucl. Eng. Des. 208, 29-50 (2001). https://doi.org/10.1016/ S0029-5493(01)00351-X

E. Epifano, C. Guéneau, R.C. Belin, R. Vauchy et al., Inorg. Chem. 56(13), 7416-7432 (2017). https://doi.org/ 10.1021/acs.inorgchem.7b00572

European Cooperation for Space Standardisation-ECSS, ECSS-E-HB-11A (2017). https://ecss.nl/home/ ecss-e-hb-11a-technology-readiness-level-trl-guidelines-1-march-2017/, accessed 30 August 2019

European Space Agency (ESA), Safety Policy on the Use of Nuclear Power Sources, United Nations Office for Outer Space Affairs, Committee on the Peaceful Uses of Outer Space, Scientific and Technical Subcommittee, Fifty-sixth session. Paper A/AC.105/C.1/2019/CRP.10 (2019)

J.A. Fay, F.R. Riddell, J. Aeronaut. Sci. 25(2), 54 (1958)

G. Gilley, M. Crook, T. Bradshaw et al., in International Cryocooler Conference Proceedings (2018). https://cryocooler.org/resources/Documents/C20/387.pdf, accessed 11 November 2019

S.F. Hoerner, Fluid Dynamic Drag: Practical Information on Aerodynamic Drag and Hydrodynamic Resistance, Brick Town, New Jersey (1965)

T.C. Holgate et al., J. Electron. Mater. (2014). https://doi.org/10.1007/s11664-014-3564-9 
G. Hula (ed.), Idaho National Laboratory, US Department of Energy, Atomic Power in Space II: A History (2015). https://www.inl.gov/research-program/space-power-systems/, accessed 30 August 2019

H. Inouye et al., Oak Ridge National Lab Report, ORNL-4813, UC-33 Propulsion and Energy Conversion (1972). https://www.osti.gov/servlets/purl/4633320, accessed 30 August 2019

R.I. Jaffee et al., Refractory Metals and Alloys, vol. 11 (1961), pp. 383-464

E.W. Johnson, Monsanto Research Corporation, Mound Laboratory Report, MLM-3250(OP) (1984). https://www.osti.gov/servlets/purl/5688898, accessed 30 August 2019

R.D. Klett, Sandia Laboratory Report, SC-RR-64-2141 (1964). https://www.osti.gov/servlets/purl/4630398, accessed 30 August 2019

R.J.M. Konings, O. Beneš, A. Kovács et al., J. Phys. Chem. Ref. Data 43(1), 013101 (2014). https://doi.org/ $10.1063 / 1.4825256$

D.P. Kramer, R.M. Ambrosi, E.J. Watkinson et al. (American Nuclear Society), in Proceedings of Nuclear and Emerging Technologies for Space (NETS 2018), Paper 24209 (2018). 978-0-89448-744-6

M. Lacoste, A. Lacombe, P. Joyez et al., Acta Astronaut. 50(6), 357 (2002). https://doi.org/10.1016/ S0094-5765(01)00178-3

F. Lebreton, R.C. Belin, T. Delahye et al., J. Solid State Chem. 196, 217 (2012). https://doi.org/10.1016/ j.jssc.2012.06.027

A. Masters, N. Achilleos, C.B. Agnor et al., Planet. Space Sci. 104, 108-121 (2014)

P. McLure, in A Presentation to: The National Academies of Sciences, Engineering, and Medicine Committee on Biological and Physical Sciences in Space, Los Alamos National Lab (2018). http://sites.nationalacademies.org/cs/groups/ssbsite/documents/webpage/ssb_185452.pdf, accessed 30 August 2019

Mersen, https://www.mersen.com/products/graphite-specialties/carboncarbon-composite/carbon-carboncomposite-grades, accessed 30 August 2019

R. Mesalam, H.R. Williams, R.M. Ambrosi et al., Appl. Energy 226, 1208-1218 (2018). https://doi.org/ 10.1016/j.apenergy.2018.05.041

R. Mesalam, H.R. Williams, R.M. Ambrosi et al., AIP Adv. 9(5), 055006 (2019). https://doi.org/ 10.1063/1.5095619

T. Nishi, A. Itoh, K. Ichise, Y. Arai, J. Nucl. Mater. 414(2), 109-113 (2011). https://doi.org/10.1016/ j.jnucmat.2011.01.019

H. Okamoto, et al., Bi-Te (Bismuth-Tellurium), Binary Alloy Phase Diagrams, ed. by T.B. Massalski, 2nd edn. (1990), pp. 800-801

A.A. Pustovalov, in Proceedings of the 5th European conference on thermoelectrics, Odessa, Ukraine, 10-12 September (2007). http://ect2007.its.org/ect2007.its.org/system/files/u1/pdf/05.pdf, accessed 5 December 2019

Z.R. Putnam, R.D. Braun, J. Guid. Control Dyn. 38(3), 414 (2015)

D.M. Rowe, Thermoelectrics Handbook: Macro to Nano (CRC Taylor and Francis, Boca Raton, 2006), pp. 710

C. Sari, E. Zamorani, J. Nucl. Mater. 37(3), 324-330 (1970). https://doi.org/10.1016/0022-3115(70)90162-5

M. Sarsfield, C. Campbell, C. Carrigan et al., E3S Web Conf. 16, 05003 (2017). https://doi.org/10.1051/ e3sconf/20171605003

M. Sarsfield, J. Brown, C. Campbell et al., Prog. Nucl. Energy 106, 396-416 (2018). https://doi.org/ 10.1016/j.pnucene.2018.02.008

W.W. Schulz, The Chemistry of Americium. ERDA Critical Review Series (1976). https://www.osti.gov/ servlets/purl/7232133, accessed 30 August 2019

R.E. Tate LANL Report, LA-9078-MS (1982). https://www.osti.gov/servlets/purl/5253319, accessed 30 August 2019

C. Thiriet, R.J.M. Konings, J. Nucl. Mater. 320(3), 292-298 (2003). https://doi.org/10.1016/ S0022-3115(03)00188-0

D.J. Trump, Presidential Memorandum on Launch of Spacecraft Containing Space Nuclear Systems (2019). https://www.whitehouse.gov/presidential-actions/presidential-memorandum-launch-spacecraftcontaining-space-nuclear-systems/, accessed 30 August 2019

J-F. Vigier, D. Freis, P. Pöml et al., Inorg. Chem. 578, 4317-4327 (2018). https://doi.org/10.1021/ acs.inorgchem. $7 \mathrm{~b} 03148$

E.J. Watkinson, Ph.D. Thesis "Space Nuclear Power Systems: Enabling Innovative Space Science and Exploration Missions" (2017). http://hdl.handle.net/2381/40461

E.J. Watkinson, C.E. Whiting, C.D. Barklay et al. (American Nuclear Society), in Proceedings of Nuclear and Emerging Technologies for Space Conference, Paper 5041 (2015)

E.J. Watkinson, R.M. Ambrosi, H.R. Williams et al., J. Nucl. Mater. 486, 308-322 (2017a). https://doi.org/ 10.1016/j.jnucmat.2017.01.011 
E.J. Watkinson, R.M. Ambrosi, D.P. Kramer et al., J. Nucl. Mater. 491, 18-30 (2017b). https://doi.org/ 10.1016/j.jnucmat.2017.04.028

E.J. Watkinson, D. Chateigner, R.M. Ambrosi et al., J. Nucl. Mater. 507, 158-163 (2018). https://doi.org/ 10.1016/j.jnucmat.2018.05.003

E.J. Watkinson, R. Ambrosi, D. Freis et al., in Proceedings IEEE Aerospace Conference (2019). https:// doi.org/10.1109/AERO.2019.8741815

H.R. Williams, R.M. Ambrosi, N.P. Bannister et al., Int. J. Energy Res. (2011). https://doi.org/10.1002/ er. 1864

H.R. Williams, H. Ning, M.J. Reece, R.M. Ambrosi et al., J. Nucl. Mater. 433(1-3), 116-123 (2013). https://doi.org/10.1016/j.jnucmat.2012.09.030

H.R. Williams, R.M. Ambrosi, K. Chen et al., J. Alloys Compd. 626, 368-374 (2014)

B.G. Woods, L.C. Arnold, T.S. Balint, AIP Conf. Proc. 813, 348 (2006). https://doi.org/10.1063/1.2169212

Y. Yamada et al., Metrologia 38, 213-219 (2001). https://doi.org/10.1088/0026-1394/38/3/3 